\title{
Neutrino pair annihilation above merger remnants: implications of a long-lived massive neutron star
}

\author{
A. Perego, H. Yasin, A. Arcones \\ Institut für Kernphysik, Technische Universität Darmstadt, Schlossgartenstraße 2, \\ 64289 Darmstadt, Germany. \\ GSI Helmholtzzentrum für Schwerionenforschung GmbH, Planckstraße 1, 64291 \\ Darmstadt, Germany. \\ E-mail: albino.perego@physik.tu-darmstadt.de
}

December 2016

\begin{abstract}
Binary neutron star mergers are plausible progenitor candidates for short gamma-ray bursts (GRBs); however, a detailed explanation of their central engine is still lacking. The annihilation of neutrino pairs has been proposed as one of the possible powering mechanisms. We present calculations of the energy and momentum deposition operated by neutrino pair annihilation above merger remnants. Starting from the results of a detailed, three-dimensional simulation of the aftermath of a binary neutron star merger, we compute the deposition rates over a time scale comparable to the life time of the disk $(t \approx 0.4 \mathrm{~s})$, assuming a long-lived massive neutron star (MNS). We model neutrino emission using a spectral leakage scheme and compute the neutrino annihilation rates using a ray-tracing algorithm. We find that the presence of the MNS increases the energy deposition rate by a factor $\sim 2$, mainly due to the annihilation of radiation coming from the MNS with radiation coming from the disk. We compute the impact of relativistic effects and discover that, despite they can significantly change the local rate intensity, the volume-integrated results are only marginally decreased. The cumulative deposited energy, extrapolated to $1 \mathrm{sec}$, is $\approx 2.2 \times 10^{49} \mathrm{erg}$. A comparison with the inferred short GRB energetics reveals that in most cases this energy is not large enough, even assuming small jet opening angles and a long-lived MNS. Significantly more intense neutrino luminosities (a factor 5-10 larger) are required to explain most of the observed short GRB. We conclude that it is unlikely that neutrino pair annihilation can explain the central engine of short GRBs alone.
\end{abstract}

Keywords: accretion disks, gamma-ray burst, neutrinos, relativistic processes, stars: neutron.

\section{Introduction}

Binary neutron star (BNS) mergers are the catastrophic fate of relativistic binary systems formed by a pair of neutron stars (NSs). The intense emission of gravitational waves (GW) that characterizes the final phases of the inspiral and its expected 
frequencies of $\sim 1-10^{3} \mathrm{~Hz}$ make merging BNSs one of the primary targets of ground based GW detectors, such as advanced LIGO, advanced VIRGO, and, in the nearby future, KAGRA (e.g., Aasi et al. 2015, Acernese et al. 2015, Aso et al. 2013). In addition, these events are expected to inject matter and energy into the interstellar medium, and to produce a rich variety of paramount astrophysical phenomena, including the nucleosynthesis of the heaviest elements (e.g., Lattimer et al. 1977, Symbalisty \& Schramm 1982, Freiburghaus et al. 1999, Korobkin et al. 2012, Goriely et al. 2011, Bauswein, Goriely \& Janka 2013, Hotokezaka et al. 2013, Wanajo et al. 2014, Martin et al. 2015, Radice et al. 2016, Wu et al. 2016), and the emission of characteristic electromagnetic transients from the freshly synthesized radioactive nuclei (e.g., Li \& Paczyński 1998, Metzger et al. 2010, Roberts et al. 2011, Tanaka \& Hotokezaka 2013, Bauswein, Goriely \& Janka 2013, Metzger \& Fernández 2014, Grossman et al. 2014), and radio flares from the expanding ejecta (e.g., Nakar \& Piran 2011, Margalit \& Piran 2015).

A BNS merger is expected to leave behind a compact object surrounded by a hot and dense accretion disk. Inside this remnant, gravitational energy is efficiently converted to internal energy and emitted in the form of neutrinos. The nature of the central object depends primarily on intrinsic attributes of the merging system (such as the NSs masses and their ratio) and on the still uncertain properties of hot nuclear matter above nuclear saturation density. The direct collapse of the merging NSs into a black hole $(\mathrm{BH})$ is foreseen only for very massive colliding objects (e.g., Bauswein, Baumgarte \& Janka 2013), while in most cases the production of a (possibly metastable) differentially rotating massive neutron star (MNS) is expected. If the MNS mass exceeds the maximum allowed NS mass, the MNS will collapse to a $\mathrm{BH}$ on a characteristic time scale. The duration of this phase depends sensitively on several parameters and poorly understood processes happening inside the MNS. Among them, we recall the amount of angular momentum and its distribution, the effects of thermal support and the consequences of neutrino cooling, the role and the properties of magnetic fields.

The large amount of energy available in these events, together with the short time scale and the reduced volume that characterize the merger and its aftermath, led to the hypothesis that BNS mergers represent (together with the merger of BH-NS binaries) a plausible progenitor system for short gamma-ray bursts (GRBs) at cosmological distances (e.g., Paczynski 1986, Narayan et al. 1992, Woosley 1993, Ruffert \& Janka 1999, Rosswog et al. 2003). Observational evidences collected over the last years seem to support this hypothesis (e.g., Berger 2014, Fong et al. 2015, Ghirlanda et al. 2016). However, a satisfactory and detailed explanation of how the merger of a compact binary system involving at least one NS powers a relativistic jet is still missing and the central engine of short GRBs still remains veiled. Several different mechanisms have been suggested. Among them, the conversion of energy by magnetohydrodynamical effects in BH-torus systems (Blandford \& Znajek 1977, Paschalidis et al. 2015, Dionysopoulou et al. 2015), the extraction of magnetic energy from highly magnetized, long-lived MNS (Metzger et al. 2011), and the annihilation of neutrino-antineutrino pairs in low density regions above the remnant (Eichler et al. 1989). The conditions needed for the formation 
and collimation of a relativistic jet above a merger remnant, as well as the interactions with the accretion disk, non-relativistic winds and dynamic ejecta, have been recently investigated with increasing details and accuracy (Aloy et al. 2005, Murguia-Berthier et al. 2014, Nagakura et al. 2014, Duffell et al. 2015, Just et al. 2016, Murguia-Berthier et al. 2016).

Neutrino emission from merger remnants has been the topic of several dedicated studies (Ruffert et al. 1997, Di Matteo et al. 2002, Rosswog \& Liebendörfer 2003, Setiawan et al. 2004, Setiawan et al. 2006, Chen \& Beloborodov 2007, Dessart et al. 2009, Caballero et al. 2012, Janiuk et al. 2013, Perego et al. 2014, Just et al. 2015, Foucart et al. 2015). The annihilation of neutrino-antineutrino pairs above BH-torus systems has been extensively studied, also considering the effects of general relativity on the neutrino propagation (Jaroszynski 1993, Popham et al. 1999, Asano \& Fukuyama 2000, Miller et al. 2003, Kneller et al. 2006, Birkl et al. 2007, Zalamea \& Beloborodov 2011, Just et al. 2016). Annihilation rates above MNS-disk system have been computed by Dessart et al. (2009) and Richers et al. (2015). In the former case, several snapshots from the first $100 \mathrm{~ms}$ of an axisymmetric merger aftermath simulation were post-processed using both a $S_{n}$ transport scheme and a simpler gray leakage scheme to model neutrino radiation. In the latter, four snapshots taken from long-term, axisymmetric simulations of merger aftermaths, and covering the 0-3 sec interval were post-processed with a Monte Carlo radiative transfer code and, again, with a simpler gray leakage scheme.

In this work, we compute the energy and momentum deposition rates above BNS merger remnant over a time scale of $400 \mathrm{~ms}$, comparable with the life time of the disk. We post-process several merger configurations taken from a long-term, three dimensional simulation of the aftermath of a BNS merger. We assume a long-lived MNS and specifically investigate the impact of the neutrino emission from the MNS. We also explore the impact of relativistic effects in the neutrino propagation on the annihilation rates, in the presence of a MNS. Finally, we discuss our results in connection with the energy inferred from short GRB observations, to address the question whether neutrino pair annihilation can be responsible for the production of relativistic jets above BNS mergers. The paper is structured as follows: in Section 2, we present the merger aftermath simulation from which we take the remnant configuration and the neutrino emission properties. Our method to compute the annihilation rates, both in a Newtonian

and in a relativistic framework, is outlined in Section 3. Section 4 is devoted to the presentation of the annihilation calculations while in Section 5 we analyze the impact of a long lived-MNS on the annihilation rates. Our results are compared with short GRB observations in Section 6. We summarize and conclude in Section 7.

\section{Model of binary NS merger remnant}

Our annihilation rate calculations are based on the numerical results of a three dimensional, Newtonian simulation of the aftermath of a binary neutron star merger 
under the influence of neutrino cooling and heating (Perego et al. 2014). The initial conditions for our model were taken from a high-resolution SPH simulation of the merger of two equal mass $\left(1.4 M_{\odot}\right)$ neutron stars (Price \& Rosswog 2006). We mapped the remnant configuration at $\sim 20 \mathrm{~ms}$ after the merger inside our equally spaced Cartesian grid. At this time, the remnant was composed by an almost axisymmetric, stationary rotating MNS with a mass of $M_{\mathrm{NS}} \approx 2.6 \mathrm{M}_{\odot}$, surrounded by a thick accretion disk with a mass of $M_{\text {disk }} \approx 0.18 \mathrm{M}_{\odot}$. We employed a rather stiff nuclear equation of state (EOS), HS(TM1) (Hempel et al. 2012), for consistency with the initial conditions. The radius of the MNS, defined as the radial coordinate where the matter density drops below $5 \times 10^{12} \mathrm{~g} \mathrm{~cm}^{-3}$, was located in a range between $17 \mathrm{~km}$ and $25 \mathrm{~km}$ along the vertical and the equatorial directions, respectively. Neutrinos of all flavors were emitted from the remnant with a total luminosity larger than $10^{53} \mathrm{erg} \mathrm{s}^{-1}$. The re-absorption of a fraction of the emitted neutrinos inside the disk caused the formation of a neutrino-driven wind on a time scale of a few tens of milliseconds. At the same time, matter expanded from the disk along the equatorial direction. This was the beginning of the so-called disk evaporation (Fernández \& Metzger 2013, Just et al. 2015). The wind developed mainly from the disk and the funnel above the central neutron star has a lower density than the material extending in the equatorial direction. We evolved the system for $\sim 400 \mathrm{~ms}$. Since our uniform spatial resolution $(\delta x=1 \mathrm{~km})$ was not sufficient to properly resolve the MNS, we assumed the region inside $\mathcal{S}$ as stationary, where $\mathcal{S}$ is an oblate spheroid of $z$ as symmetry axis, center in the domain origin, semi-axis $a=30 \mathrm{~km}$ along $x$ and $y$, and $b=23 \mathrm{~km}$ along $z$, and matter density above $5 \times 10^{11} \mathrm{~g} \mathrm{~cm}^{-3}$. Profiles of the matter density and electron fraction inside the MNS and in the innermost part of the disk at $40 \mathrm{~ms}$ inside our simulation are shown in Figure 1, together with the incipient neutrino-driven wind.

Neutrino physics is modeled using an energy dependent (spectral) leakage scheme (Perego et al. 2014, Perego et al. 2016). All neutrino quantities are binned logarithmically in energy, with twelve energy bins between $2 \mathrm{MeV}$ and $200 \mathrm{MeV}$. The neutrino reactions included in the calculations are the emission, absorption, and scattering off free nucleons. Neutrino pair emission from electron-positron annihilation and nucleon-nucleon bremsstrahlung are also included in optically thick conditions, as well as absorption from their inverse reactions. We model three independent neutrino species, $\nu_{e}, \bar{\nu}_{e}$, and $\nu_{x}$, the latter being a collective species for $\mu$ and $\tau$ (anti)neutrinos. Spectral optical depths, $\tau_{\nu}$, are computed by minimizing the line integral of inverse mean free paths along several possible straight propagation directions. This allows the distinction between optically thin $\left(\tau_{\nu} \lesssim 1\right)$ and optically thick $\left(\tau_{\nu} \gg 1\right)$ regions. We further distinguish between scattering, $\tau_{\nu, \mathrm{sc}}$, and energy optical depths, $\tau_{\nu \text {,en }}$ (Raffelt 2001). We denote the neutrino surfaces as the surfaces where $\tau_{\nu}=2 / 3$. In the case of $\tau_{\nu, \text { sc }}$ they correspond to the last scattering surfaces. In the case of $\tau_{\nu \text {,en }}$ they locate the transition from a thermally coupled to a non-thermally coupled regime. The position of the scattering and energy surfaces inside the remnant at $40 \mathrm{~ms}$ in our simulation are represented in Figure 2, for all neutrino species and for five representative 

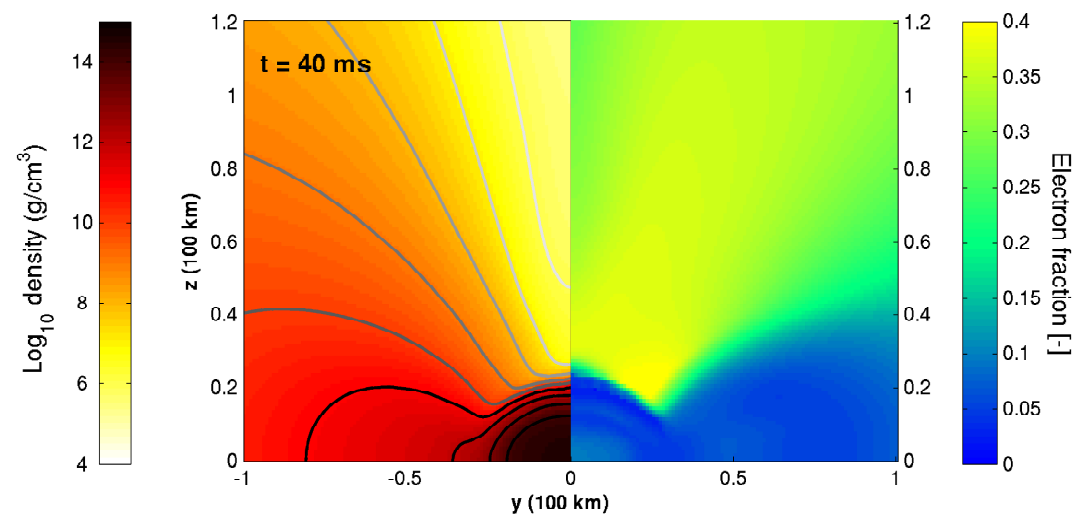

Figure 1. Vertical slice ( $x=0$ plane) of the three dimensional domain for the matter density (left) and the electron fraction (right) at $40 \mathrm{~ms}$ of our simulation. The solid lines on the density profile represent isodensity contours, ranging from $10^{14} \mathrm{~g} \mathrm{~cm}^{-3}$ (black line) to $10^{6} \mathrm{~g} \mathrm{~cm}^{-3}$ (light gray line). The densest part of the remnant corresponds to MNS, while density contours of $10^{11} \mathrm{~g} \mathrm{~cm}^{-3}$ and $10^{10} \mathrm{~g} \mathrm{~cm}^{-3}$ track the outer boundary of the disk. The expanding matter located above the remnant and characterized by low density and reduced neutron richness corresponds to the developing neutrino-driven wind.
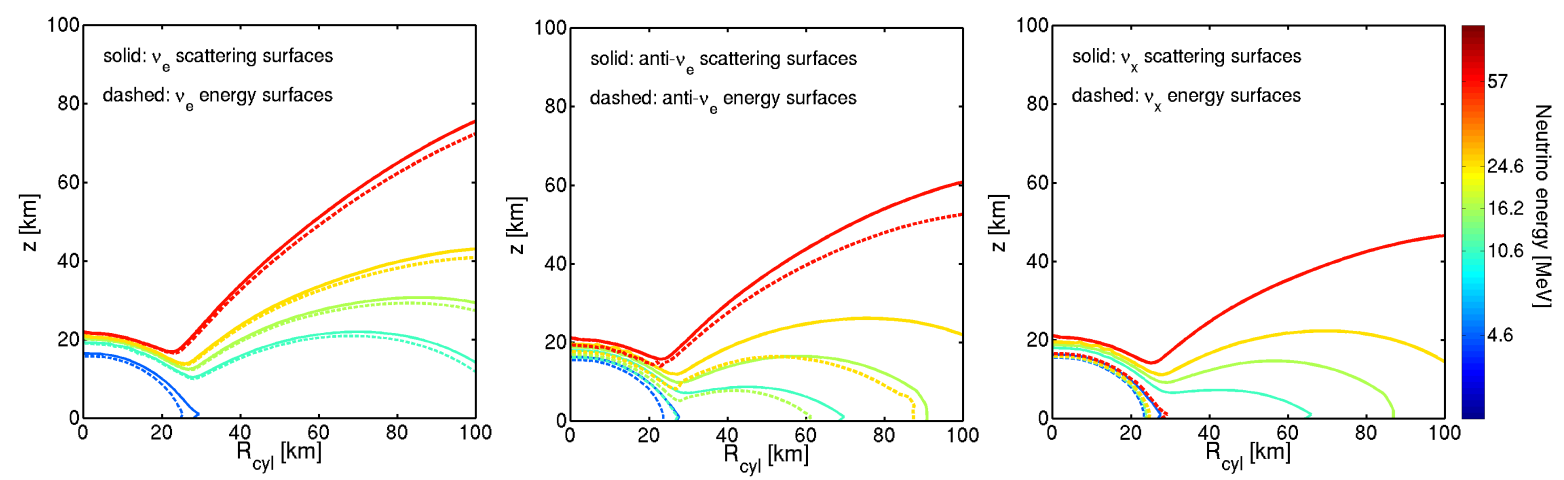

Figure 2. Neutrino scattering (solid lines) and energy (dashed lines) surfaces at $40 \mathrm{~ms}$ in the simulation. The three panels correspond to $\nu_{e}$ (left), $\bar{\nu}_{e}$ (center) and $\nu_{x}$ (right), while lines with different colors and increasing thickness represent increasing neutrino energies.

energies. Neutrinos are considered emitted isotropically in the half space denoted by $\mathbf{n}_{\tau}=-\nabla \tau_{\nu, \mathrm{sc}} /\left|\nabla \tau_{\nu, \mathrm{sc}}\right|$, both from the neutrino scattering surfaces (for radiation diffusing from optically thick conditions) and from their production site (for radiation emitted in optically thin conditions) (Perego et al. 2014, appendix).

\subsection{Neutrino luminosities}

We review the most relevant properties of the neutrino emission obtained from our hydrodynamical simulation, since they represent a key ingredient for the calculations of the annihilation rates. In Figure 3, we present the evolution of the neutrino luminosities $\left(L_{\nu}\right.$ left panel) and root mean squared (RMS) energies $\left(\epsilon_{\mathrm{RMS}, \nu} \equiv \sqrt{\left\langle\epsilon_{\nu}^{2}\right\rangle}\right.$, right panel) 

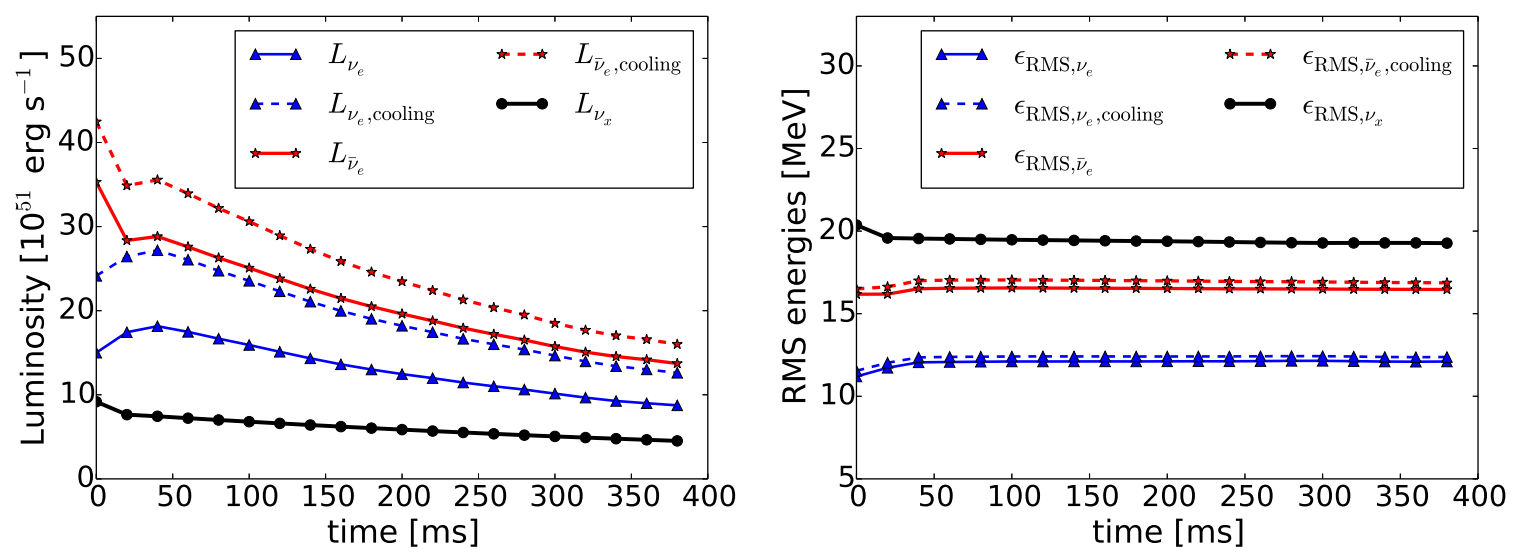

Figure 3. Evolution of the neutrino total luminosities (left) and root mean squared energies (RMS, right). Blue lines (triangles) refer to $\nu_{e}$, red lines (stars) to $\bar{\nu}_{e}$, black lines (circles) to $\nu_{x}$. All quantities are measured at infinity. Solid and dashed lines refer to net and cooling luminosities (or RMS energies), respectively. In the former case neutrino absorption processes outside the last scattering surfaces are included; in the latter, not.

for all the three independent neutrino species, over the entire simulation time. Both quantities are measured at infinity, taking into account that a fraction of the emitted neutrinos (especially $\nu_{e}$ 's and, less significantly, $\bar{\nu}_{e}$ 's) get absorbed on their way out. To show the effect of neutrino absorption outside the neutrino surfaces, we plot also the luminosities and RMS energies obtained including the neutrinos emitted at the last scattering surfaces and outside, but assuming the neutrino absorptivity to be zero for $\tau_{\nu, \mathrm{sc}}<2 / 3$. We call them "cooling" luminosities and RMS energies (dashed lines).

Neutrino luminosities are powered by the cooling of the remnant and by the process of accretion inside the disk. After an initial phase $(t<40 \mathrm{~ms})$ when the disk dissipates internal perturbations and transients, and the total luminosity slightly increases, all the luminosities decrease with time. The RMS neutrino energies reflect the different depths and thermodynamical conditions at which neutrinos thermally decouple from matter. While the values obtained for $\nu_{e}$ and $\bar{\nu}_{e}$ are consistent with the ones reported in the literature, the values obtained for $\nu_{x}$ are smaller than expected (by $\sim 20 \%$ ). As reported in Perego et al. (2014), this discrepancy is due to the lack of spatial resolution in our simulation deep inside the MNS, where the thermal decoupling of $\nu_{x}$ 's occurs.

Due to the stationary treatment of the MNS, the luminosities coming from the spheroid $\mathcal{S}, L_{\nu, \mathcal{S}}$,sim, are practically constant during the simulation. To mimic the decrease produced by the cooling process, we assume the evolution of the luminosities coming from the innermost part of the computational domain as an exponential decline:

$$
L_{\nu, \mathcal{S}}(t)=L_{\nu, \mathcal{S}, \operatorname{sim}} \exp \left(-t / t_{\text {cool }}\right)
$$

in analogy to the luminosity produced by a cooling proto-NS (Fischer et al. 2010, Hüdepohl et al. 2010). The cooling time scale $t_{\text {cool }}$ is estimated as $t_{\text {cool }} \approx \Delta E_{\mathcal{S}} / L_{\nu, \mathcal{S} \text {,sim }}$, where $\Delta E_{\mathcal{S}}$ is the thermal component of the internal energy inside $\mathcal{S}$, calculated from 
the initial remnant configuration. From our simulation we obtained $t_{\text {cool }} \approx 0.725 \mathrm{~s}$.

\section{Calculation of the annihilation rates}

\subsection{Newtonian calculation}

We consider the annihilation of neutrino pairs into electron positron pairs, i.e. $\nu_{i}+\bar{\nu}_{i} \rightarrow$ $e^{+} e^{-}$with $i \in\{e, \mu, \tau\}$. We calculate the energy and momentum deposition rates due to this process outside the most relevant neutrino surfaces, post-processing the input data obtained in our simulation of the aftermath of a binary NS merger. Since the power associated with neutrino-antineutrino annihilation is expected to be subdominant compared with the one of charged-current neutrino absorption reactions, we can safely compute the former in a post-processing step (Dessart et al. 2009). For consistency with the underlying simulation, we start computing the rate in a Newtonian framework. Moreover, we assume the neutrino to be massless and we presently do not include the effects of neutrino oscillations in our analysis (e.g., Malkus et al. 2016, Zhu et al. 2016, Frensel et al. 2016). We calculate the local energy deposition rate $q_{\nu_{i}, \bar{\nu}_{i}}(\mathbf{x}, t)$ for the neutrino flavor $i$ according to the following expression (Kneller et al. 2006, Dessart et al. 2009, and references therein):

$$
\begin{aligned}
q_{\nu_{i}, \bar{\nu}_{i}}(\mathbf{x}, t)= & \frac{\sigma_{0}\left(c_{A}^{2}+c_{V}^{2}\right)_{\nu_{i}, \bar{\nu}_{i}}}{6 c\left(m_{e} c^{2}\right)^{2}} \int_{\Omega_{\nu_{i}}} \mathrm{~d} \Omega_{\nu_{i}} \int_{\Omega_{\bar{\nu}_{i}}} \mathrm{~d} \Omega_{\bar{\nu}_{i}} \int_{0}^{\infty} \mathrm{d} \epsilon_{\nu_{i}} \int_{0}^{\infty} \mathrm{d} \epsilon_{\bar{\nu}_{i}} \\
& \left(\epsilon_{\nu_{i}}+\epsilon_{\bar{\nu}_{i}}\right) I_{\nu_{i}} I_{\bar{\nu}_{i}}(1-\cos \Phi)^{2},
\end{aligned}
$$

We extend the previous formula to compute the deposited momentum $\mathbf{p}_{\nu_{i}, \bar{\nu}_{i}}$ :

$$
\begin{aligned}
\mathbf{p}_{\nu_{i}, \bar{\nu}_{i}}(\mathbf{x}, t)= & \frac{\sigma_{0}\left(c_{A}^{2}+c_{V}^{2}\right)_{\nu_{i}, \bar{\nu}_{i}}}{6 c^{2}\left(m_{e} c^{2}\right)^{2}} \int_{\Omega_{\nu_{i}}} \mathrm{~d} \Omega_{\nu_{i}} \int_{\Omega_{\bar{\nu}_{i}}} \mathrm{~d} \Omega_{\bar{\nu}_{i}} \int_{0}^{\infty} \mathrm{d} \epsilon_{\nu_{i}} \int_{0}^{\infty} \mathrm{d} \epsilon_{\bar{\nu}_{i}} \\
& \left(\epsilon_{\nu_{i}} \mathbf{n}_{\nu_{i}}+\epsilon_{\bar{\nu}_{i}} \mathbf{n}_{\bar{\nu}_{i}}\right) I_{\nu_{i}} I_{\bar{\nu}_{i}}(1-\cos \Phi)^{2},
\end{aligned}
$$

In Eqs. (2) and (3), $c$ is the speed of light, $m_{e}$ the mass of the electron, $\sigma_{0}=$ $4 m_{e}^{2} G_{F}^{2} / \pi \hbar^{4} \approx 1.71 \times 10^{-44} \mathrm{~cm}^{2}$ the typical neutrino cross section, with $G_{F}$ the Fermi constant, and $\hbar$ the reduced Planck constant. Furthermore, $I_{\nu}=I_{\nu}\left(\mathbf{x}, t, \epsilon_{\nu}, \mathbf{n}_{\nu}\right)$ denotes the radiation intensity at position $\mathbf{x}$ and time $t$, for neutrinos with energy $\epsilon_{\nu}$ along the direction $\mathbf{n}_{\nu}$. The infinitesimal solid angle and energy in the momentum space of the propagating radiation is represented by $\mathrm{d} \Omega_{\nu}$ and $\mathrm{d} \epsilon_{\nu}$, respectively. The cosine of the angle between the momenta of the colliding neutrinos is given by $\cos \Phi=\left(\mathbf{n}_{\nu} \cdot \mathbf{n}_{\bar{\nu}}\right)$. Equations (2) and (3) apply to all neutrino species, provided the appropriate values of the weak coupling constants $c_{A}$ and $c_{V}=c_{A}+2 \sin ^{2} \theta_{W}$, where $\sin ^{2} \theta_{W}=0.23$. In the case of $\nu_{e}$ and $\bar{\nu}_{e}, c_{A}=1 / 2$, while for $\nu_{x}$ and $\bar{\nu}_{x}, c_{A}=-1 / 2$. Note that in the expressions for $q_{\nu_{i}, \bar{\nu}_{i}}$ and $\mathbf{p}_{\nu_{i}, \bar{\nu}_{i}}$ we have neglected phase space blocking for $e^{ \pm}$in the final state, as well as a term proportional to $\left(m_{e} c^{2}\right)^{2} /\left(\epsilon_{\nu_{i}} \epsilon_{\bar{\nu}_{i}}\right)$ in the integrand. In fact, the latter is $10^{3}$ times smaller for typical neutrino energies (e.g., Perego et al. 2014).

Since the neutrino intensities required in Eqs. (2) and (3) are not directly provided by our simulation, we compute them based on the local emissivities. We fix one point 
$B$ outside the most relevant neutrino surfaces. We denote the amount of energy $\mathrm{d} E_{B}$ transported across a surface element $\mathrm{d} A_{B}$ in a time $\mathrm{d} t$ by $\mathrm{d} N_{B}$ neutrinos in an energy interval $\mathrm{d} \epsilon_{B}$ around $\epsilon_{B}$ propagating inside a solid angle $\mathrm{d} \Omega_{B}$ around $\mathbf{n}_{B}$, in terms of radiation intensity $I_{B} \equiv I_{\nu}\left(\mathbf{x}_{B}, \epsilon_{B}, \mathbf{n}_{B}\right)$ as $\mathrm{d} E_{B}=\epsilon_{B} \mathrm{~d} N_{B}=I_{B} \mathrm{~d} A_{B} \mathrm{~d} t \mathrm{~d} \Omega_{B} \mathrm{~d} \epsilon_{B}$. We further express the amount of energy emitted at any point $A$ by a volume $\mathrm{d} V_{A}$ in a time $\mathrm{d} t$ in $\mathrm{d} N_{A}$ neutrinos with an energy interval $\mathrm{d} \epsilon_{A}$ around $\epsilon_{A}$ and into a solid angle $\mathrm{d} \Omega_{A}$ around $\mathbf{n}_{A}$, as $\mathrm{d} E_{A}=\epsilon_{A} \mathrm{~d} N_{A}=\eta_{A} \mathrm{~d} V_{A} \mathrm{~d} t \mathrm{~d} \Omega_{A} \mathrm{~d} \epsilon_{A}$, where $\eta_{A} \equiv \eta_{\nu}\left(\mathbf{x}_{A}, \epsilon_{A}, \mathbf{n}_{A}\right)$ is the local emissivity. Conservation of particles and energy implies:

$$
\epsilon_{B} \mathrm{~d} N_{B}=\epsilon_{A} \mathrm{~d} N_{A} \exp \left(-\Delta \tau_{\mathrm{en}, A B}\right),
$$

where we took into account that a fraction of the emitted neutrinos get absorbed on their way to $B$. This amount is estimated using an exponential damping based on the difference of the energy optical depth $\tau_{\text {en }}$ between $A$ and $B, \Delta \tau_{\text {en, } A B}=\tau_{\text {en, } A}-\tau_{\text {en }, B}$. Inserting the definitions of intensity and emissivity into Eq. (4), and rewriting $d A_{B}=$ $d_{A B}^{2} d \Omega_{A}$, where $d_{A B}$ denoted the distance between the two points, we obtain:

$$
I_{B} d \Omega_{B} d \epsilon_{B}=\frac{\eta_{A} d V_{A} d \epsilon_{A}}{d_{A B}^{2}} \exp \left(-\Delta \tau_{\mathrm{en}, A B}\right) .
$$

We model the emission of neutrinos from the neutrino surfaces and from the optically transparent regions consistently with the treatment used in the post-merger simulation. Specifically,

$$
\eta_{\nu}\left(\mathbf{x}_{A}, \epsilon_{A}, \mathbf{n}_{A}\right)=\left\{\begin{array}{lr}
\text { const. } & \text { if }\left\langle\mathbf{n}_{A}\right\rangle \cdot \mathbf{n}_{A}>0 \\
0 & \text { otherwise }
\end{array}\right.
$$

i.e., we assume that radiation is emitted isotropically in the half space identified by the unitary vector $\left\langle\mathbf{n}_{A}\right\rangle$ and we take $\left\langle\mathbf{n}_{A}\right\rangle=\mathbf{n}_{\tau, A}$ (see Section 2).

We discretize the three dimensional space with cylindrical coordinates $\left\{R_{i}, \phi_{j}, z_{k}\right\}$ where $i, j$ and $k$ can vary between 1 and $N_{R}, N_{\phi}$ and $N_{z}$, respectively. For the neutrino energy, we use the same energy bins that we employed in our post-merger simulation, $\left\{\epsilon_{m}\right\}_{m=1, N_{e}}$, with $N_{e}=12$. From the results of our simulation, we extract cylindrically averaged values for the spectral optical depths $\left(\tau_{\nu}\right)_{i, k, m} \equiv \tau_{\nu}\left(R_{i}, z_{k}, \epsilon_{m}\right)$ and for the (solid angle integrated) neutrino emission rates in each energy bin $\epsilon_{m}$, $\left(R_{\nu}\right)_{i, k, m} \equiv R_{\nu}\left(R_{i}, z_{k}, \epsilon_{m}\right)$. Integrating Eq. (6) over the whole solid angle, we can express $\eta_{\nu}$ in terms of $R_{\nu}$ :

$$
\left(R_{\nu}\right)_{i, k, m}=\frac{2 \pi\left(\eta_{\nu}\right)_{i, k, m} \mathrm{~d} \epsilon_{m}}{\epsilon_{m}}
$$

If we discretize Eq. (2) according to

$$
\begin{aligned}
& q_{\nu_{i}, \bar{\nu}_{i}}\left(\mathbf{x}_{B}, t\right)=\frac{\sigma_{0}\left(c_{A}^{2}+c_{V}^{2}\right)_{\nu_{i}, \bar{\nu}_{i}}}{6 c\left(m_{e} c^{2}\right)^{2}} \sum_{A(i, j, k)} \sum_{A^{\prime}\left(i^{\prime}, j^{\prime}, k^{\prime}\right)} \sum_{m, m^{\prime}}\left(\epsilon_{m}+\epsilon_{m^{\prime}}\right) \\
& \left(\mathrm{d} \Omega_{\nu} \mathrm{d} \epsilon_{\nu} I_{\nu}\right)_{A(i, j, k, m) \rightarrow B}\left(\mathrm{~d} \Omega_{\bar{\nu}} \mathrm{d} \epsilon_{\bar{\nu}} I_{\bar{\nu}}\right)_{A^{\prime}\left(i^{\prime}, j^{\prime}, k^{\prime}, m^{\prime}\right) \rightarrow B}\left(1-\cos \Phi_{A, A^{\prime}}\right)^{2},
\end{aligned}
$$


inserting Eqs. (5) and (7) we obtain

$$
\begin{aligned}
& q_{\nu_{i}, \bar{\nu}_{i}}\left(\mathbf{x}_{B}, t\right)=\frac{\sigma_{0}\left(c_{A}^{2}+c_{V}^{2}\right)_{\nu_{i}, \bar{\nu}_{i}}}{6 c\left(m_{e} c^{2}\right)^{2}} \sum_{A(i, j, k)} \sum_{A^{\prime}\left(i^{\prime}, j^{\prime}, k^{\prime}\right)} \sum_{m, m^{\prime}}\left(\epsilon_{m}+\epsilon_{m^{\prime}}\right) \epsilon_{m} \epsilon_{m}^{\prime} \\
& \Theta\left(\left\langle\mathbf{n}_{A, m}\right\rangle \cdot \mathbf{n}_{A B}\right) \Theta\left(\left\langle\mathbf{n}_{A^{\prime}, m^{\prime}}\right\rangle \cdot \mathbf{n}_{A^{\prime} B}\right) \frac{\left(R_{\nu}\right)_{i, j, k, m} \mathrm{~d} V_{i, j, k}}{2 \pi d_{A B}^{2}} \frac{\left(R_{\bar{\nu}}\right)_{i^{\prime}, j^{\prime}, k^{\prime}, m^{\prime}} \mathrm{d} V_{i^{\prime}, j^{\prime}, k^{\prime}}}{2 \pi d_{A^{\prime} B}^{2}} \\
& \exp \left(-\left(\left(\Delta \tau_{\nu, \mathrm{en}}\right)_{A B, m}+\left(\Delta \tau_{\nu, \mathrm{en}}\right)_{A^{\prime} B, m^{\prime}}\right)\right)\left(1-\cos \Phi_{A, A^{\prime}}\right)^{2},
\end{aligned}
$$

where $\mathrm{d} V_{i, j, k}$ is the volume element in cylindrical coordinates, $\mathbf{n}_{A^{(\prime)} B}=\left(\mathbf{x}_{B}-\mathbf{x}_{\left.A^{(\prime)}\right)} / \mid \mathbf{x}_{B}-\right.$ $\mathbf{x}_{A^{(\prime)}} \mid$, and $\Theta$ is the Heaviside step function. For the discrete version of Eq. (3), it is enough to replace $\left(\epsilon_{m}+\epsilon_{m}^{\prime}\right)$ with $\left(\epsilon_{m} \mathbf{n}_{A B}+\epsilon_{m}^{\prime} \mathbf{n}_{A^{\prime} B}\right) / c$ in Eq. (9).

Starting from the local expression of the energy deposition rate, Eq. (2), we compute the volume-integrated energy deposition rate, $Q_{\nu_{i}, \bar{\nu}_{i}}(t)$, as

$$
Q_{\nu_{i}, \bar{\nu}_{i}}(t)=\int_{\tilde{V}} q_{\nu_{i}, \bar{\nu}_{i}}(t, \mathbf{x}) \mathrm{d} V
$$

Following Birkl et al. (2007), we define $\tilde{V}$ as the volume outside the most relevant neutrino surfaces, and in which the radial component of the total momentum deposited by neutrinos is pointing outwards. In addition, we designate the annihilation efficiency as (e.g., Eichler et al. 1989, Just et al. 2016):

$$
\eta_{\nu, \bar{\nu}}=Q_{\nu, \bar{\nu}} /\left(L_{\nu}+L_{\bar{\nu}}\right)
$$

Since the integrand in Eq. (10) is proportional to $\left(c_{A}^{2}+c_{V}^{2}\right)_{\nu_{i}, \overline{\nu_{i}}}\left(\epsilon_{\nu_{i}}+\epsilon_{\bar{\nu}_{i}}\right) I_{\nu_{i}} I_{\bar{\nu}_{i}}$, it is natural to assume that (e.g., Goodman et al. 1987, Janka 1991, Setiawan et al. 2006):

$$
Q_{\nu_{i}, \bar{\nu}_{i}} \approx \frac{\sigma_{0}\left(c_{A}^{2}+c_{V}^{2}\right)_{\nu_{i}, \bar{\nu}_{i}}}{96 \pi^{2} c\left(m_{e} c^{2}\right)^{2}} L_{\nu_{i}} L_{\bar{\nu}_{i}}\left[\frac{\left\langle\epsilon_{\nu_{i}}^{2}\right\rangle}{\left\langle\epsilon_{\nu_{i}}\right\rangle} G_{\nu_{i}, \bar{\nu}_{i}}+\frac{\left\langle\epsilon_{\bar{\nu}_{i}}^{2}\right\rangle}{\left\langle\epsilon_{\bar{\nu}_{i}}\right\rangle} H_{\nu_{i}, \bar{\nu}_{i}}\right]
$$

where $G_{\nu_{i}, \bar{\nu}_{i}}$ and $H_{\nu_{i}, \bar{\nu}_{i}}$ are geometrical factors that contain the integral of all the residual spatial and geometrical dependencies. In the next sections, we will test the validity of this parametrization, based on detailed numerical results, using both the luminosities at infinity and the cooling luminosities.

Finally, we define $E_{\nu_{i}, \bar{\nu}_{i}}(t)$ as the deposited cumulative energy:

$$
E_{\nu_{i}, \bar{\nu}_{i}}(t)=\int_{0}^{t} Q_{\nu_{i}, \bar{\nu}_{i}}\left(t^{\prime}\right) \mathrm{d} t^{\prime}
$$

alongside with a global annihilation efficiency,

$$
\bar{\eta}_{\nu, \bar{\nu}}=E_{\nu_{i}, \bar{\nu}_{i}} / \int_{0}^{t}\left(L_{\nu}+L_{\bar{\nu}}\right) \mathrm{d} t^{\prime} .
$$

\subsection{Relativistic calculation}

The hydrodynamics simulation and the annihilation rate calculations presented in the previous sections are performed in a Newtonian framework. However, matter in the disk moves close to Keplerian, and typical orbital speeds are given by:

$$
\frac{v_{\phi}(R)}{c} \sim \sqrt{\frac{G M_{\mathrm{NS}}}{R c^{2}}} \approx 0.309\left(\frac{M_{\mathrm{NS}}}{2.6 M_{\odot}}\right)^{1 / 2}\left(\frac{R_{\mathrm{NS}}}{20 \mathrm{~km}}\right)^{-1 / 2}\left(\frac{R}{2 R_{\mathrm{NS}}}\right)^{-1 / 2}
$$


where $M_{\mathrm{NS}}$ and $R_{\mathrm{NS}}$ are the MSN mass and radius, respectively. Moreover, the ratio between the the Schwarzschild radius $R_{g}$ and the actual MNS radius is

$$
\frac{R_{g}}{R_{\mathrm{NS}}}=\frac{2 G M_{\mathrm{NS}}}{R_{\mathrm{NS}} c^{2}} \approx 0.382\left(\frac{M_{\mathrm{NS}}}{2.6 M_{\odot}}\right)\left(\frac{R_{\mathrm{NS}}}{20 \mathrm{~km}}\right)^{-1} .
$$

Thus, relativistic effects due to the fast motion of matter and to the intense gravitational field are potentially relevant. A consistent relativistic calculation requires to start from the results of relativistic hydrodynamical simulations of a neutron star merger, and to compute the annihilation rates in a relativistic framework. Due to the Newtonian character of our input simulations, this is not entirely possible. However, we can compute the impact of special and general relativistic effects on the neutrino propagation and on the annihilation process using our Newtonian input data. We consider the following effects on the propagation from the emission to the interaction points: the Doppler and beaming effects due to the fast motion of the emitting fluid elements, and the redshift and light bending effects due to motion of the radiation in the gravitational potential. A detailed explanation of the adopted spacetime model, as well as of the implementation of the relativistic effects is presented in Appendix A.

Following Birkl et al. (2007) and Zalamea \& Beloborodov (2011), we compute the volume-integrated energy deposition rates in the local frames

$$
Q_{\nu_{i}, \bar{\nu}_{i}}^{\mathrm{rel}}(t)=\int_{\tilde{V}} q_{\nu_{i}, \bar{\nu}_{i}}(t, \mathbf{x}) \sqrt{\operatorname{det}\left(g_{a b}\right)} \mathrm{d} r \mathrm{~d} \theta \mathrm{d} \phi,
$$

as well as the energy deposition rates measured by an infinitely distant observer:

$$
Q_{\nu_{i}, \bar{\nu}_{i}}^{\mathrm{rel}, \infty}(t)=\int_{\tilde{V}} q_{\nu_{i}, \bar{\nu}_{i}}(t, \mathbf{x}) \sqrt{\operatorname{det}\left(g_{\mu \nu}\right)} \mathrm{d} r \mathrm{~d} \theta \mathrm{d} \phi,
$$

where the redshift due to the gravitational field is also included $\ddagger$. Each of these two rates can be integrated over time to obtain the cumulative energies, $E_{\nu_{i}, \bar{\nu}_{i}}^{\mathrm{rel}}(t)$ and $E_{\nu_{i}, \bar{\nu}_{i}}^{\mathrm{rel}, \infty}(t)$.

\subsection{Numerical setup}

We perform the calculation of the annihilation rates for twenty equally spaced time steps, ranging from 0 to $380 \mathrm{~ms}$. Since the input data are cylindrically symmetric, we expect also $q_{\nu_{i}, \bar{\nu}_{i}}$ and $\mathbf{p}_{\nu_{i}, \bar{\nu}_{i}}$ to be axisymmetric. Thus, we compute the rates only on the $\phi=11 \pi / 45$ plane and we assume them to be valid for any polar angle $\phi$. For the discretization of the $\phi$ angle required by our calculations, we choose $N_{\phi}=45\left(\Delta \phi=8^{\circ}\right)$ and we test the convergence of our results with respect to this parameter $\left(\delta Q_{\nu_{i}, \overline{\nu_{i}}} \lesssim 3 \%\right.$ for $N_{\phi}=60$ ). To save computing time, we consider the emission of neutrinos only from cells with $\rho \geq 2 \times 10^{9} \mathrm{~g} \mathrm{~cm}^{-3}$. Also in this case, this simplification introduces an error lower, at most, than a few percents. 

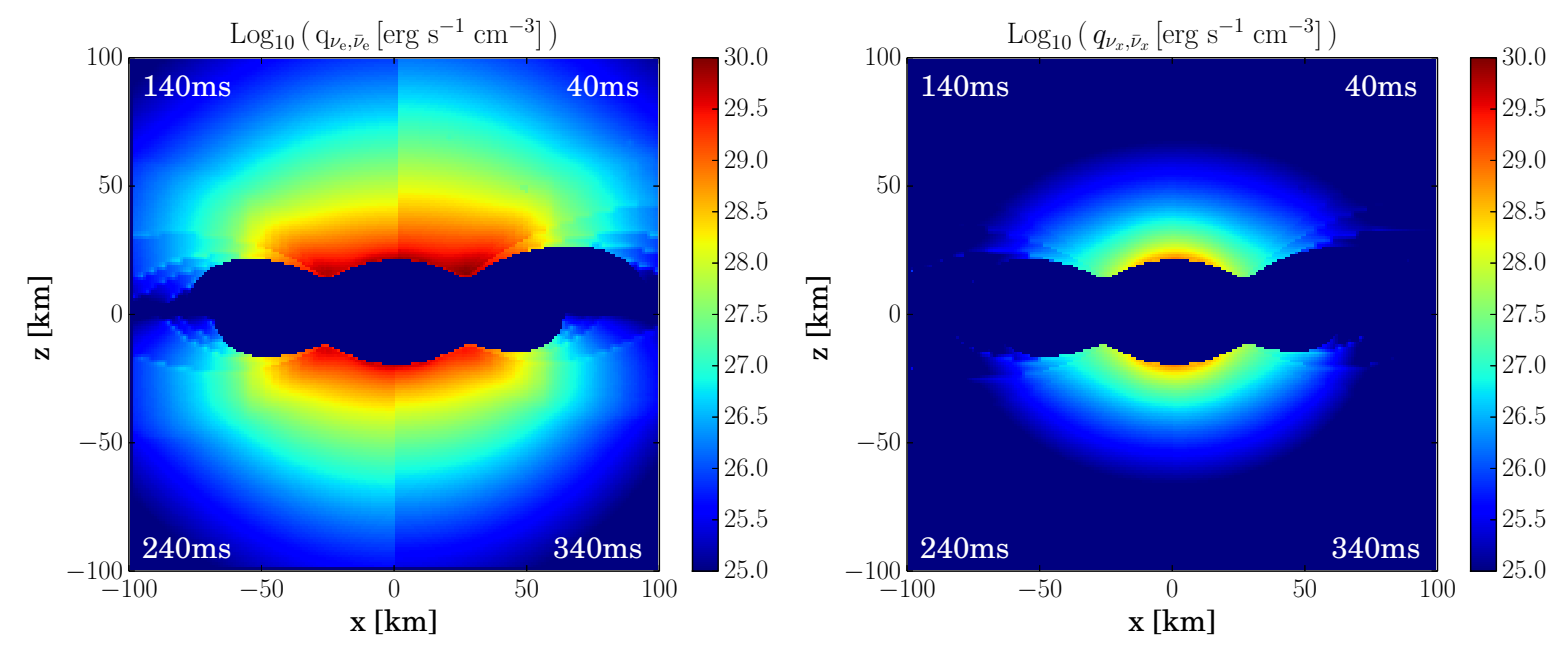

Figure 4. Color coded is the local energy deposition rate $q_{\nu, \bar{\nu}}$, as a function of the cylindrical coordinates, in the $\phi=11 \pi / 45$ plane and in the intervals $[0,100] \mathrm{km} \times$ $[0,100] \mathrm{km}$. In the left panel we plot the rate for $\nu_{e}, \bar{\nu}_{e}$, in the right one for $\nu_{x}, \bar{\nu}_{x}$. The four quadrants refer to different times, as indicated by the labels. If $\rho>10^{11} \mathrm{~g} \mathrm{~cm}^{-3}$ we set the rates to the minimum (blue area).

\section{Energy and momentum: deposition rates}

In Figure 4, we show the local energy deposition rate $q_{\nu_{i}, \bar{\nu}_{i}}(\mathbf{x}, t)$ at four different times, both for electron flavor (left panel) and heavy flavor (right panel) neutrino pairs. These rates have been obtained using our standard (i.e., non-relativistic) approach. We notice that in both cases and at each time the energy deposition rate is more intense close to the remnant and decreases for increasing distances, as a consequence of the decrease of the neutrino intensity. Moreover, even if the rates decrease with time due to the decline of the neutrino luminosities, their spatial distribution remains qualitatively similar during the evolution of the system. However, the results obtained for the different flavors differ significantly. In the $\nu_{e}-\bar{\nu}_{e}$ case, the energy deposition is more intense and distributed over a wider volume, including the regions above the MNS and the innermost part of the disk. In the $\nu_{x}-\bar{\nu}_{x}$ case, the energy deposition is concentrated above the MNS and the decrease of $q_{\nu_{x}, \bar{\nu}_{x}}(\mathbf{x}, t)$ with time is less pronounced, due to the milder reduction of the $\nu_{x}$ luminosities.

The deposition rate for the linear momentum is presented in Figure 5, again for four different times. The arrows indicate the direction of $\mathbf{p}_{\nu, \bar{\nu}}$ projected on the vertical plane. Due to the axisymmetry of the neutrino emissivities, we expect the azimuthal component of the deposited momentum to be negligible. The geometry of the MNS and of the disk influences the emission direction of the neutrinos and, in turn, the direction of $\mathbf{p}_{\nu, \bar{\nu}}$, according to Eq. (3). In particular, the MNS produces an almost spherical emission inside the funnel above its surface. This combines with the axisymmetric emission from $\ddagger$ Note that Eqs. (17) and (18) differ by the indexes of the metric tensor: in the former expression only the spatial part of $g_{\mu \nu}$ is considered, while the full metric is used in the latter. 

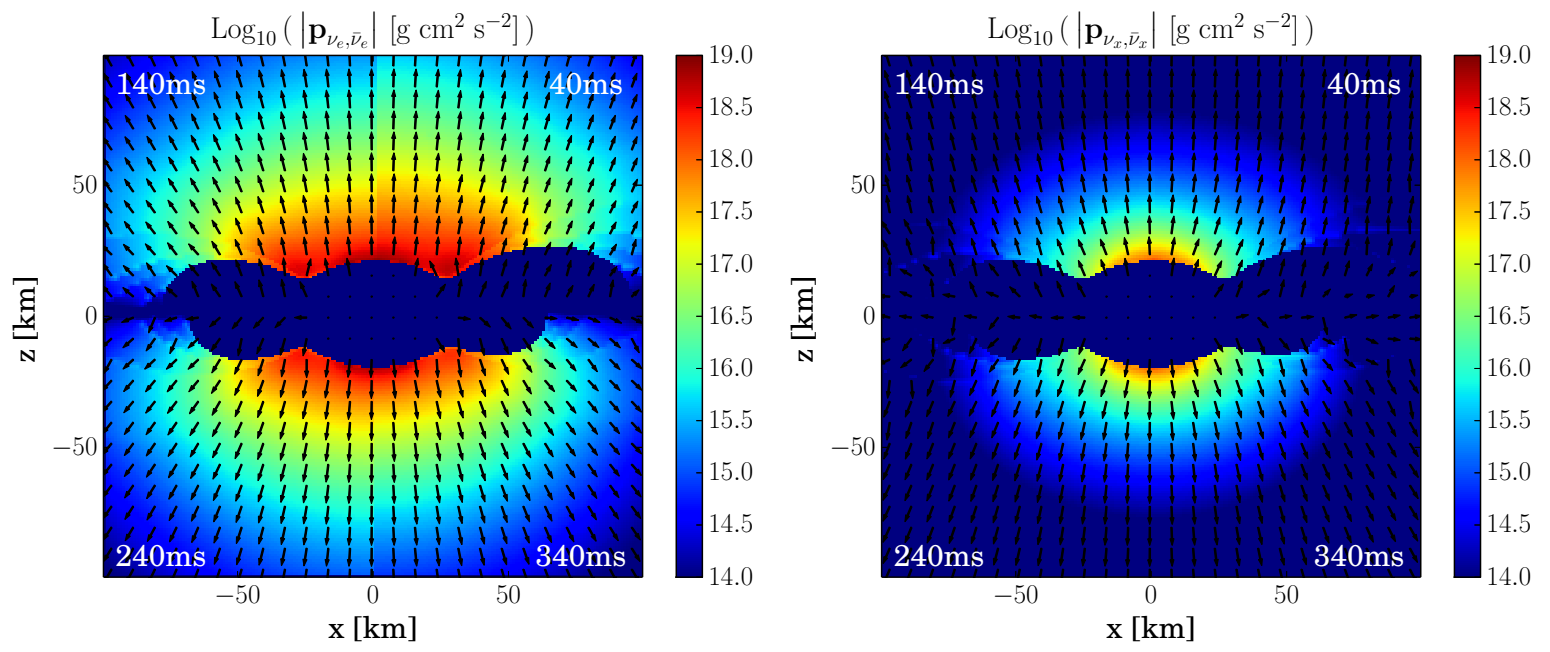

Figure 5. Same as in Figure 4, but for the momentum deposition rate $\mathbf{p}_{\nu, \bar{\nu}}$. The color code indicate the modulus of the vector, $\left|\mathbf{p}_{\nu, \bar{\nu}}\right|$, while the arrow the direction projected on the plane. Due to the symmetry of the neutrino sources, $\left(p_{\nu, \bar{\nu}}\right)_{\phi} \approx 0$.
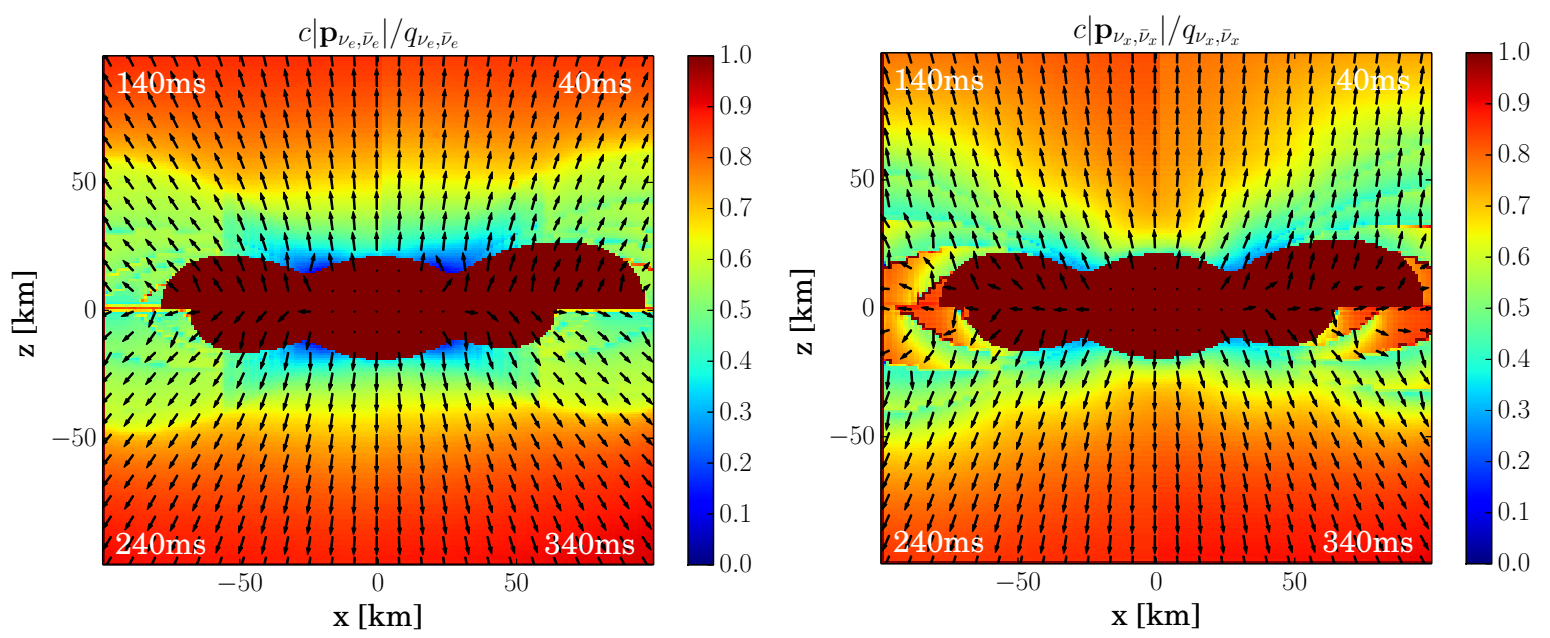

Figure 6. Same as in Figure 5, but for the ratio between the modulus of the momentum deposition rate, $\left|\mathbf{p}_{\nu, \bar{\nu}}\right|$, times $c$ and the energy deposition rate, $q_{\nu, \bar{\nu}}$. If $\rho>10^{11} \mathrm{~g} \mathrm{~cm}^{-3}$ we set the ratio to the maximum (red area).

the disk. The latter happens predominantly at the transition between the MNS and the disk, where neutrinos are emitted towards the funnel and, on average, perpendicularly to the neutrino surfaces (see Figure 2). At larger radii, the average emission direction from the disk becomes parallel to the $z$ axis or even pointing outwards. Since in the case of $\nu_{x}$ the neutrino emission comes mainly from the MNS, $\mathbf{p}_{\nu_{x}, \bar{\nu}_{x}}$ presents a more radial structure. We notice that at larger times, when the disk has shrunk its radius and consumed its mass, $\mathbf{p}_{\nu_{e}, \bar{\nu}_{e}}$ also develops into a more radial configuration.

A comparison between the energy and momentum deposition reveals that the intensity of the latter tracks the one of the former. However, the dependence on the colliding angle $\Phi$ contained in Eqs. (2) and (3), and the vector sum that characterizes the 

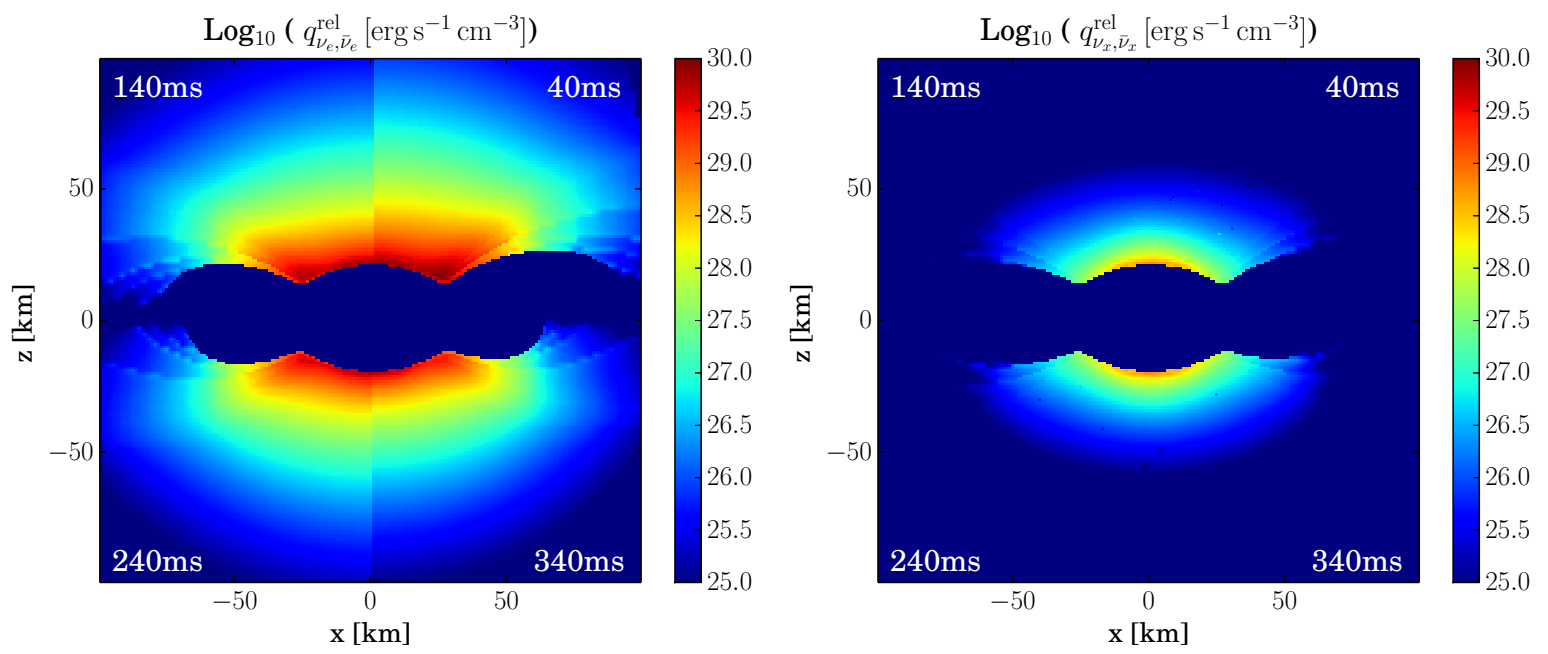

Figure 7. Same as in Figure 4, but including the special and general relativistic effects for the neutrino propagation outside the neutrino surfaces.
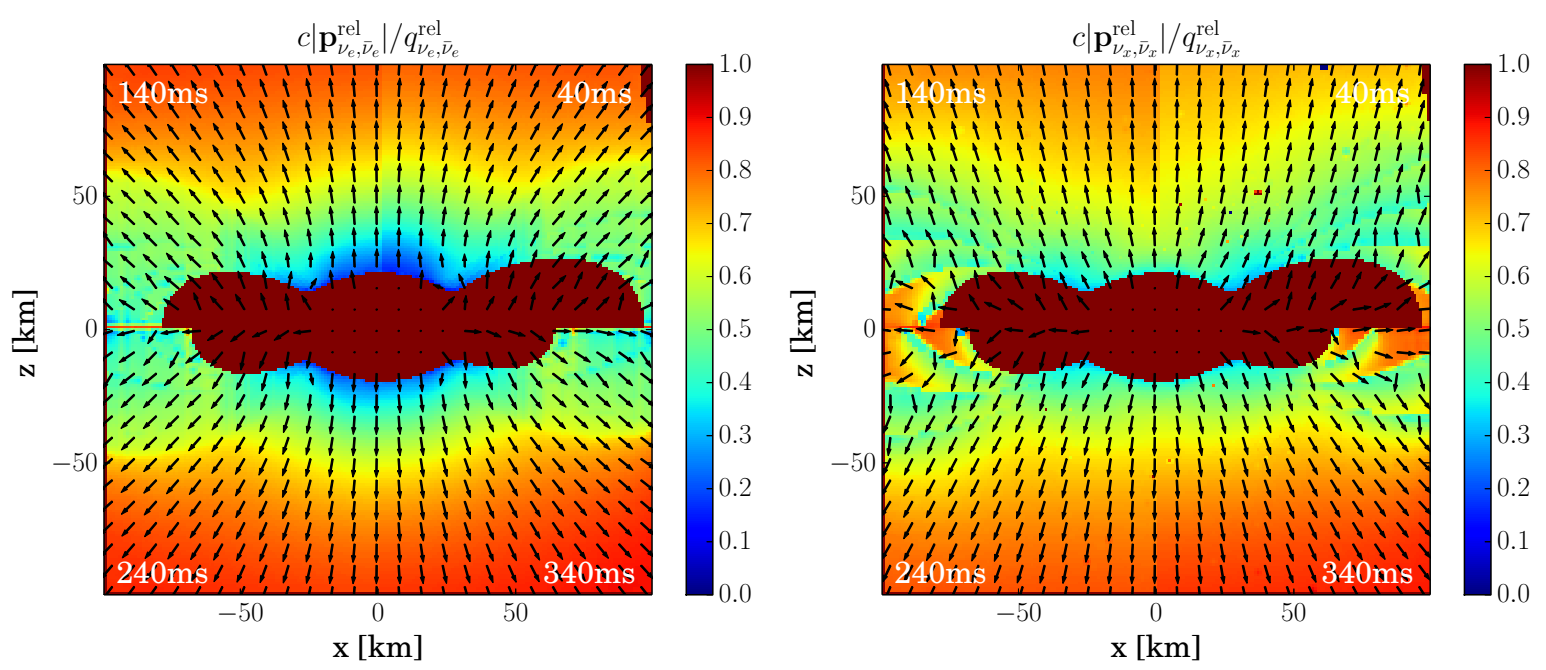

Figure 8. Same as in Figure 6 but including the special and general relativistic effects for the neutrino propagation outside the neutrino surfaces.

momentum deposition imply that $c\left|\mathbf{p}_{\nu, \bar{\nu}}\right|<q_{\nu, \bar{\nu}}$. In particular, the more isotropic and symmetric the local neutrino distribution, the less efficient is the momentum deposition compared to the energy one. In Figure 6 , we present $c\left|\mathbf{p}_{\nu, \bar{\nu}}\right| / q_{\nu, \bar{\nu}}$ and confirm that close to the MSN and to the disk, where the propagation directions are expected to be more isotropic, the ratio is significantly smaller than 1 , reaching even 0.2 , while it increases up to 0.8 at larger distances.

We repeat the pair annihilation calculations including special and general relativistic effects, and we present the results for the local energy deposition rate in Figure 7. The inclusion of the relativistic effects does not change the qualitative features of the energy deposition rate. A careful comparison between the energy deposition rates provided by $\nu_{e}, \bar{\nu}_{e}$ pairs shows that the light bending and the gravitational blueshift increase the annihilation rates immediately above the MNS (by $\lesssim 20 \%$ ), while the 

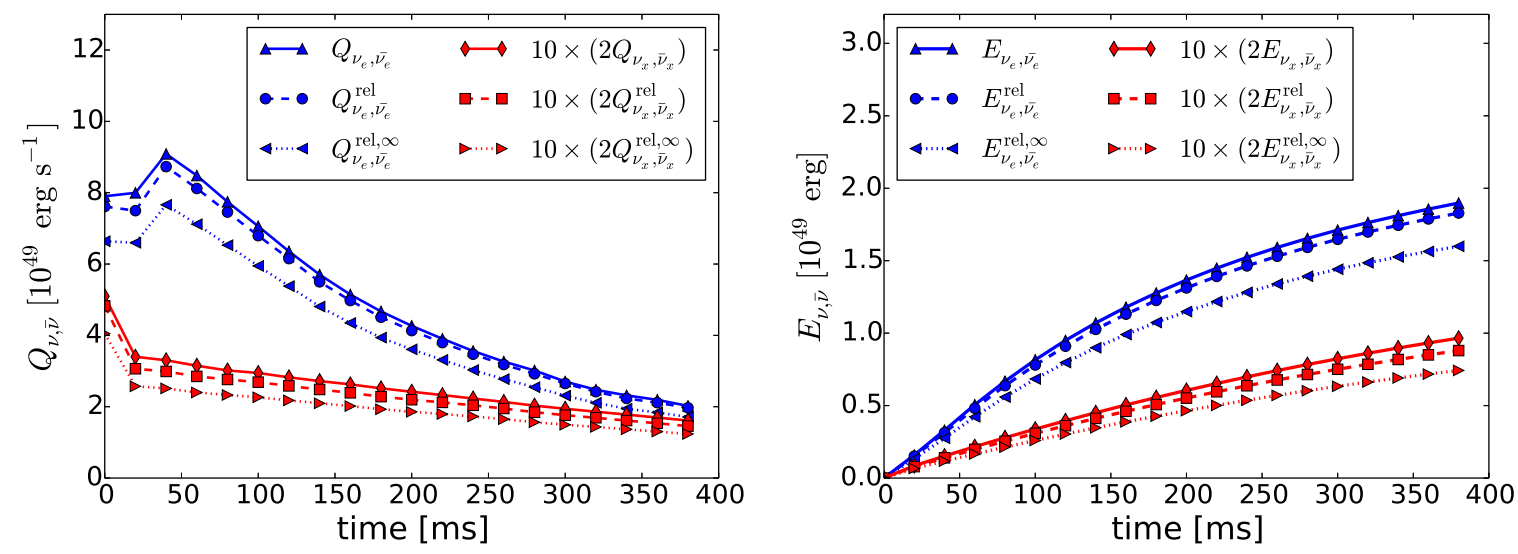

Figure 9. Volume-integrated energy rate (left) and cumulative deposited energy (right) by neutrino pair annihilation, for our reference Newtonian calculations $\left(Q_{\nu, \bar{\nu}}\right.$, $\left.E_{\nu, \bar{\nu}}\right)$ and for the calculations including relativistic effect in the neutrino propagation (local quantities, $Q_{\nu, \bar{\nu}}^{\mathrm{rel}}$ and $E_{\nu, \bar{\nu}}^{\mathrm{rel}}$, and quantities measure by a far observer $Q_{\nu, \bar{\nu}}^{\mathrm{rel}, \infty}$ and $\left.E_{\nu, \bar{\nu}}^{\mathrm{rel}, \infty}\right)$. Blue and red lines refer to electron and heavy flavor neutrinos, respectively. The factor 2 in $Q_{\nu_{x}, \bar{\nu}_{x}}$ and $E_{\nu_{x}, \bar{\nu}_{x}}$ takes into account the $\mu$ and $\tau$ flavors, while the factor 10 is only a magnification factor.

gravitational redshift, together with the beaming of the radiation emitted by matter rotating inside the disk, reduces the energy deposition rate in the funnel and above the innermost part of the disk. Where the rates are larger, the reduction is usually $\sim 20 \%$, but it increases when the distance from the MNS and the disk increases, as well as at later times. In the case of $\nu_{x}, \bar{\nu}_{x}$ pairs, the dominant effect is provided by the gravitational redshift and the result is a reduction of the energy deposition rate everywhere in the computational domain. The gain in annihilation efficiency due to the light bending is attenuated by the dominant contribution of the MNS to the $\nu_{x}$ luminosities.

The qualitative features of the momentum deposition rate computed by taking into account the relativistic effects in the neutrino propagation are very similar to the ones obtained in our reference Newtonian calculations. In Figure 8, we present the ratio between the modulus of the momentum and the energy deposition rates. A comparison with the analogue figure obtained in the Newtonian framework, Figure 6, shows that the light bending reduces the efficiency at which a net momentum is deposited more significantly, with respect to the energy deposition rate, especially close to the MNS surface and in the funnel above it.

In Figure 9, we present the volume integrated rates, $Q_{\nu_{i}, \bar{\nu}_{i}}(t)$ (left panel) and cumulative deposited energy, $E_{\nu_{i}, \bar{\nu}_{i}}(t)$ (right panel), for both the reference Newtonian calculations, Eqs. (10)-(13), and including relativistic corrections, (17). We first notice that, for the volume element in our cylindrical discretization, one has

$$
\mathrm{d} V \propto R \Delta R \Delta z .
$$

Thus, intense deposition regions located at larger cylindrical radii contribute more 
significantly than close to the rotational axis. The integration over the volume allows to quantify the difference between the different neutrino flavors: $Q_{\nu_{e}, \bar{\nu}_{e}}(t)$ is $\sim 60$ times larger than $Q_{\nu_{x}, \bar{\nu}_{x}}(t)$. Due to this large difference, the contribution of $\nu_{x}$ 's is significantly sub-dominant for the overall energy deposition process. The approximated expression (12) accounts for this considerable disparity. In particular, comparing the electron and the heavy flavors, $\left(c_{A}^{2}+c_{V}^{2}\right)_{\nu_{e}, \bar{\nu}_{e}} /\left(c_{A}^{2}+c_{V}^{2}\right)_{\nu_{x}, \bar{\nu}_{x}} \approx 4.6$ and $L_{\nu_{e}} L_{\bar{\nu}_{e}} /\left(L_{\nu_{x}} L_{\bar{\nu}_{x}}\right) \approx 12$. The inclusion of relativistic effects in the neutrino propagation changes only marginally the intensity of the energy deposition rate. In particular, the energy blueshift and light bending happening close to the MNS are globally compensated by the redshift and the light beaming in the funnel and above the disk. Overall, the integrated energy deposition measured by local observers differs only by a few percent in the Newtonian and in the relativistic case. The difference becomes more relevant $(\sim 15 \%)$ only when $Q_{\nu_{i}, \bar{\nu}_{i}}^{\mathrm{rel},}$ and $E_{\nu_{i}, \bar{\nu}_{i}}^{\mathrm{rel}, \infty}$ are considered: this is due to the inclusion of the gravitational redshift required for the deposited energy to travel from the deposition site to infinity.

The volume-integrated energy deposition rate for $\nu_{e}$ and $\bar{\nu}_{e}$ ranges between $9 \times 10^{49} \mathrm{erg} \mathrm{s}^{-1}$ and $2 \times 10^{49} \mathrm{erg} \mathrm{s}^{-1}$, with a clear decreasing trend with time. The total amount of energy deposited by neutrino pair annihilations in the funnel above the merger remnant reaches $1.95 \times 10^{49} \mathrm{erg}$ at $380 \mathrm{~ms}$ in our Newtonian calculation, while it reduces to $1.6 \times 10^{49} \mathrm{erg}$ in the relativistic calculations when the energy at infinity is considered. We notice that, even if the deposition rate has significantly decreased at the end of our simulation, this energy has not saturated yet. This is due to the longer time scales of neutrino diffusion from the MNS, $t_{\text {cool }} \sim 0.7 \mathrm{~s}$, and of disk consumption, $t_{\text {disc }} \sim 0.5 \mathrm{~s}$ (Perego et al. 2014).

\section{Role of the MNS}

In order to investigate the role of the MNS in the process of neutrino pair annihilation, we decompose the local emissivity $\eta_{\nu}$ into a contribution from the disk $\left(\eta_{\nu, \text { disk }}\right)$ and one from the neutron star $\left(\eta_{\nu, \mathrm{NS}}\right)$. All neutrinos that originate inside the spheroid $\mathcal{S}$ are tagged as "neutron star (NS) neutrinos", while all the others are identified as "disk (DS) neutrinos". We notice that this distinction refers to the origin place, but not necessarily to the emission place. While all DS neutrinos are emitted from the disk region, a fraction of the NS neutrinos can diffuse out from the disk before being emitted. This happens especially for those neutrinos emitted by the MNS along the equator.

\subsection{Neutrino luminosity and accretion rate}

In the case of an accretion disk around a $\mathrm{BH}$, the disk luminosity can be related to the accretion rate at the $\mathrm{BH}$ horizon, $L \approx \eta_{\text {acc }} \dot{M} c^{2}$, where $\eta_{\text {acc }}$ is a coefficient that quantifies the efficiency at which the rest mass of the accreting matter is converted into emitted

radiation (typically, $\eta_{\text {acc }} \lesssim 0.05$, Just et al. (2016)). Here we present the behavior of the luminosity and accretion rate based on our simulation and discuss the significant 

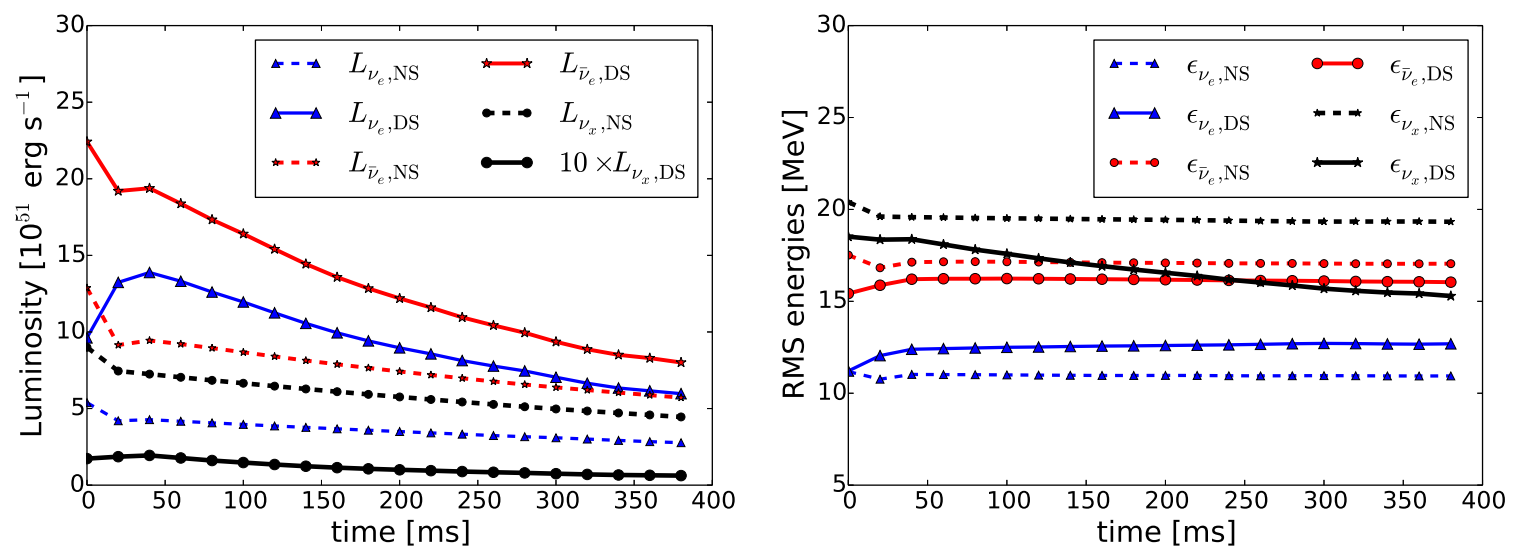

Figure 10. Disk (solid lines) and neutron star (dashed lines) contributions to the total luminosities (left) and RMS energies (right) obtained from our simulation of the aftermath of a binary neutron star merger. Blue lines (triangles) refer to $\nu_{e}$, red lines (stars) to $\bar{\nu}_{e}$, black lines (circles) to $\nu_{x}$. All quantities are measured at infinity. For this figure we used only the luminosities including the absorption of neutrinos in optically thin conditions.
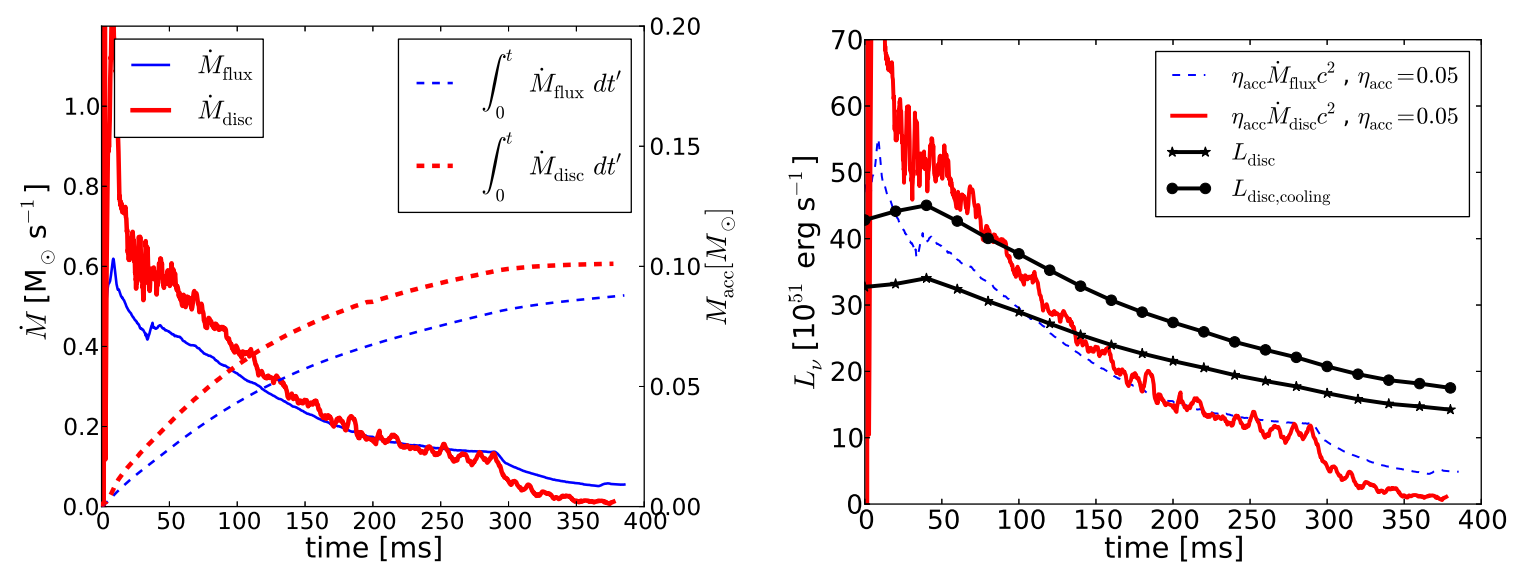

Figure 11. Left: mass accretion rates (solid lines) and cumulative accreted matter (dashed lines) computed inside the accretion disk. The red thick lines refer to the accretion rate as time derivative of the mass enclosed in the innermost part of the disk, while the blue thin lines as matter flux at a cylindrical radius of $\tilde{R}=40 \mathrm{~km}$. Right: comparison between the temporal evolution of the neutrino luminosities (black lines) and of the luminosities derived from the accretion rate, assuming $L=\eta \dot{M} c^{2}$.

differences due to the presence of a MNS.

The distinction between neutrinos coming from the MNS and from the disk allows to decompose each neutrino luminosity into a MNS and a disk contribution. In Figure 10, we show for each independent species the disk and the MNS components. The MNS contributions originate mainly from the diffusion of neutrinos from the hot and dense central remnant. Their decrease reflects the progressive cooling of the MNS and of the matter accreting on its surface. The larger disk components are more related to the accretion process and present a more pronounced reduction. 
The evolution of the accretion rate inside the disk, $\dot{M}_{\text {disk }}$ is shown in the left panel of Figure 11. Since there is no clear inner boundary where to measure the flux of the infalling matter, we compute the accretion rate as the time derivative of the mass contained inside the densest part of the disk $\left(\rho>\rho_{\min }=5 \times 10^{10} \mathrm{~g} \mathrm{~cm}^{-3}\right)$, outside a cylindrical radius $R_{\text {in }}=30 \mathrm{~km}$ :

$$
\dot{M}_{\text {disk }} \equiv-\frac{\mathrm{d} M_{\text {disk }}}{\mathrm{d} t}=-\frac{\mathrm{d}}{\mathrm{d} t} \int_{0}^{2 \pi} \int_{-\infty}^{\infty} \int_{R_{\text {in }}}^{\infty} R^{2} \rho \Theta\left(\rho-\rho_{\text {min }}\right) \mathrm{d} R \mathrm{~d} z \mathrm{~d} \phi,
$$

where $\Theta$ is the Heaviside step function. The choice of $R_{\text {in }}=30 \mathrm{~km}$ ensures the exclusion of the MNS. Since the expansion of matter in the $\nu$-driven wind and in the viscous component happen around a few times $10^{10} \mathrm{~g} \mathrm{~cm}^{-3} \lesssim \rho_{\text {min }}$, our definition of $\dot{M}_{\text {disk }}$ takes into account only the disk consumption due to the accretion process. To verify our assumption, we also compute

$$
\dot{M}_{\text {flux }} \equiv \int_{\Sigma(\tilde{R}=40 \mathrm{~km})} \rho \mathbf{v} \cdot \mathrm{d} \Sigma
$$

where $\Sigma(\tilde{R})$ is the cylindrical surface of radius $\tilde{R}$ and $\mathrm{d} \boldsymbol{\Sigma}$ is an infinitesimal surface element pointing in the positive radial direction. We choose $\tilde{R}=40 \mathrm{~km}$ since for $\mathrm{R}$ $\gtrsim 40 \mathrm{~km}$ the disk is characterized by a global infalling bulk motion along the radial direction, while at smaller radii, radially outgoing flows are also observed.

In the initial phase $(t<100 \mathrm{~ms})$, accretion onto the MNS occurs mainly from high density regions located within $\tilde{R}$, thus $\dot{M}_{\text {disk }}>\dot{M}_{\text {flux }}$. Once the disk has reached an almost stationary configuration $(t>100 \mathrm{~ms})$, the two accretion rates behave similarly and $\dot{M}_{\text {disk }} \approx \dot{M}_{\text {flux }}$. The integral of $\dot{M}_{\text {disk }}$ reveals that the MNS has accreted $\sim 0.1 M_{\odot}$ within $400 \mathrm{~ms}$.

In the right panel of Figure 11, we compare the total disk luminosity $L_{\mathrm{DS}}=$ $L_{\nu_{e}, \mathrm{DS}}+L_{\bar{\nu}_{e}, \mathrm{DS}}+4 L_{\nu_{x}, \mathrm{DS}}$ (using both the total and the cooling luminosities) with the luminosity obtained from the accretion rate, $L_{\text {acc }}=\eta_{\text {acc }} \dot{M}_{\text {disk }} c^{2}$ assuming $\eta_{\text {acc }}=0.05$. We notice that in the presence of a MNS $L_{\mathrm{DS}}$ is no more proportional to $\dot{M}$. In particular, the decline of the disk luminosity is slower than the decrease of the accretion rate. The difference with the BH case is due to the presence of the long-lived MNS, which has several consequences:

1) The MNS surface does not act as an inner, open boundary, and the transition between the MNS and the disk happens in a high density, high temperature region where the accreted matter settle on the MNS surface. The steep density gradient that characterizes this innermost part of the disk and the MNS surface translates into a large pressure gradient. While for $R \gtrsim \tilde{R} \approx 40 \mathrm{~km}$ the radial flow inside the disk is distinguished by an inward bulk motion, at smaller radii it is characterized by the presence of both inward and outward radial flows, resulting from the combination of large scale inflow, bounces on the MNS surface and fast orbital motion.

2) Even if the innermost part of the disk is moderately optically thick $\left(\tau_{\nu, \mathrm{sc}}\right) \sim 10$, matter inside it can still emit neutrinos. They diffuse out on a time scale of a few ms and are emitted at the neutrino surfaces. 

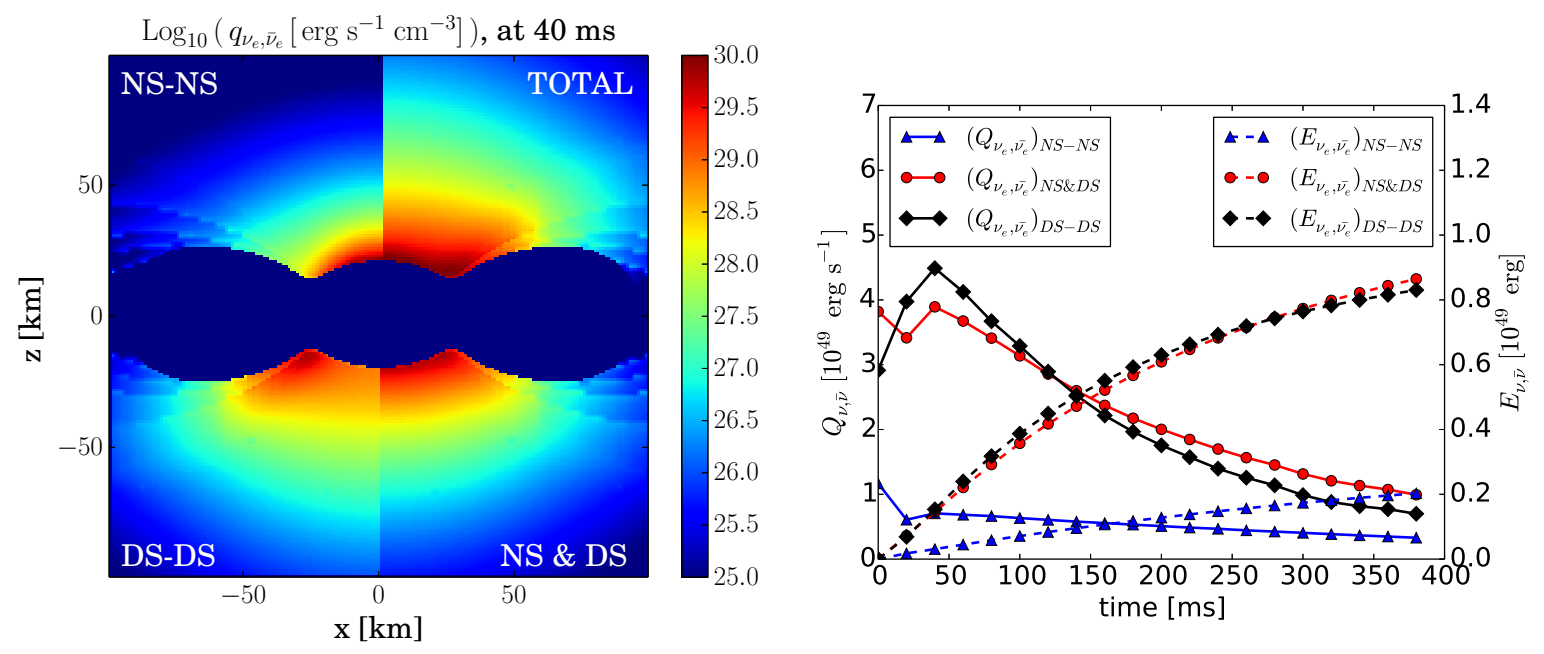

Figure 12. Left: Energy deposition rate by electron neutrino pair annihilation at $40 \mathrm{~ms}$ inside our simulation. In the four quadrants, we plot the three different contributions obtained by tagging the emitted neutrinos based on their production site (neutron star (NS), or disk (DS)) together with their sum. Right: Contributions to the volume-integrated energy rate $\left(Q_{\nu, \bar{\nu}}\right.$, solid lines) and cumulative deposited energy $\left(E_{\nu, \bar{\nu}}\right.$, dashed lines) for electron neutrino, as a function of the simulated time, coming from the NS-NS contribution (blue lines, triangles), the DS-DS contribution (black lines, diamonds), and the NS\&DS contribution (red lines, circles). For both panels, we use our standard (Newtonian) results.

3) Due to the disk finite size and consumption, the density profile inside the disk decreases and the disk becomes progressively more transparent to neutrinos. In particular, the neutrino diffusion time scale, $t_{\nu, \text { diff }} \sim \alpha_{\text {diff }} \tau_{\nu}^{2} \lambda_{\nu} / c$ where $\alpha_{\text {diff }} \sim 3$, decreases with time. At the same time, the accretion process partially compensates the internal energy emitted by the neutrino radiation. If $\epsilon_{\text {int }}$ is the specific internal energy of matter the ratio $\epsilon_{\text {int }} / t_{\nu \text {,diff }}$ increases with time in the innermost part of the disk.

In summary, in the presence of a MNS, the larger efficiency at which the internal energy can be radiated by neutrinos, together with the longer time that matter has to cool, partially compensates the disk consumption and the accretion rate attenuation.

\subsection{MNS and disk contributions to the energy deposition rate}

The splitting of the local emissivity into a neutron star and a disk component allows also the decomposition of the local radiation intensity, $I_{\nu}=I_{\nu, \mathrm{NS}}+I_{\nu, \mathrm{DS}}$. Since we deal with a pair process, the integrand in Eq. (2) for the local energy deposition rate $q_{\nu, \bar{\nu}}$ can be expanded in four different contributions according to:

$$
q_{\nu, \bar{\nu}}=\left(q_{\nu, \bar{\nu}}\right)_{\mathrm{NS}-\mathrm{NS}}+\left(q_{\nu, \bar{\nu}}\right)_{\mathrm{DS}-\mathrm{DS}}+\left[\left(q_{\nu, \bar{\nu}}\right)_{\mathrm{NS}-\mathrm{DS}}+\left(q_{\nu, \bar{\nu}}\right)_{\mathrm{DS}-\mathrm{NS}}\right] .
$$

In the left panel of Figure 12, we show the different contributions for electron flavor neutrinos at $40 \mathrm{~ms}$, alongside with their sum. In the following, we will refer to the combined neutron star-disk contribution as $\left(q_{\nu, \bar{\nu}}\right)_{\mathrm{NS} \& \mathrm{DS}} \equiv\left[\left(q_{\nu, \bar{\nu}}\right)_{\mathrm{NS}-\mathrm{DS}}+\left(q_{\nu, \bar{\nu}}\right)_{\mathrm{DS}-\mathrm{NS}}\right]$, if 
not stated differently. The different contributions deposit energy at different locations above the remnant. The NS-NS contribution is more intense immediately above the surface of the MNS and it fades rapidly for increasing radial distances. This reflects the quasi-spherical nature of the neutrino surfaces. The annihilation is efficient only close to the emission surfaces, where the intensities are larger and the cosine of the average propagation angle is $\langle\mu\rangle \sim 1 / 2$. For larger distances, the intensities decrease as $r^{-2}$ and enter the forward-peaked regimes, $\langle\mu\rangle \sim 1$ (Janka 1991). The largest energy deposition rates for the DS-DS contribution are located above the region marking the transition between the MNS and the disk. The energy rate in the funnel above the MNS is still high, but it decreases while approaching the rotational axis. This behavior points to the fact that most of the DS-DS pair annihilations happen close to the neutrino emission surfaces (for radiation emitted from contiguous zones of the disk and annihilating at all angles) rather than in the funnel, confirming results from Dessart et al. (2009). The larger intensities of the former configuration compensate for the better collision angle of the latter. Finally, for the NS\&DS contribution, neutrinos moving away from the MNS annihilate with those emitted by the disk towards the funnel. The properties of this contribution are a combination of the properties of the previous two. The results is an intense energy deposition both close to the MNS surface and above the transition region between the MNS and the disk.

We integrate the energy deposition rates over the volume for the three different contributions and the results are shown in the right panel of Figure 12. The DS-DS and the NS\&DS contributions are comparable throughout the whole simulation time. This result points out the potential relevance of a long-lived MNS, in comparison with a $\mathrm{BH}$-torus system. In fact, in the latter case, only the DS-DS contribution is expected to be present and the total energy deposited by neutrino pair annihilation can be reduced by a factor of (at least) two.

The steeper decrease of the DS-DS contribution is due to its quadratic dependence on the more rapidly decreasing disk luminosity, $q_{\mathrm{DS}-\mathrm{DS}} \propto L_{\nu, \mathrm{DS}} L_{\bar{\nu}, \mathrm{DS}}$, see Eq. (12), while for the NS\&DS contribution we expect $q_{\mathrm{NS} \& \mathrm{DS}} \propto\left(L_{\nu, \mathrm{NS}} L_{\bar{\nu}, \mathrm{DS}}+L_{\nu, \mathrm{DS}} L_{\bar{\nu}, \mathrm{NS}}\right)$. Despite the intense energy deposition associated with the NS-NS contribution in the funnel above the MNS, the volume integrated rate is significantly sub-dominant, especially at earlier times. This is a consequence of the smaller volume where the intense energy deposition occurs, see Eq. (19).

To summarize the spatial dependence of the energy deposition, in Figure 13 we present the volume integral of the energy deposited by $\nu_{e}, \bar{\nu}_{e}$ pair annihilation at three different times and in selected regions of the domain. In particular, the integration is performed only for regions where polar angles are smaller than $\theta$ or greater than $(\pi-\theta)$ (left panel), and densities are smaller than $\rho$ (right panel). The energy deposited closer to the rotational axis and at lower densities is foreseen to contribute more to the formation of a jet. A comparison between the different terms reveals that the contributions involving the MNS deposit a more significant fraction of their energy at high densities $\left(\rho>10^{11} \mathrm{~g} \mathrm{~cm}^{-3}\right)$ compared with the DS-DS contribution. However, 

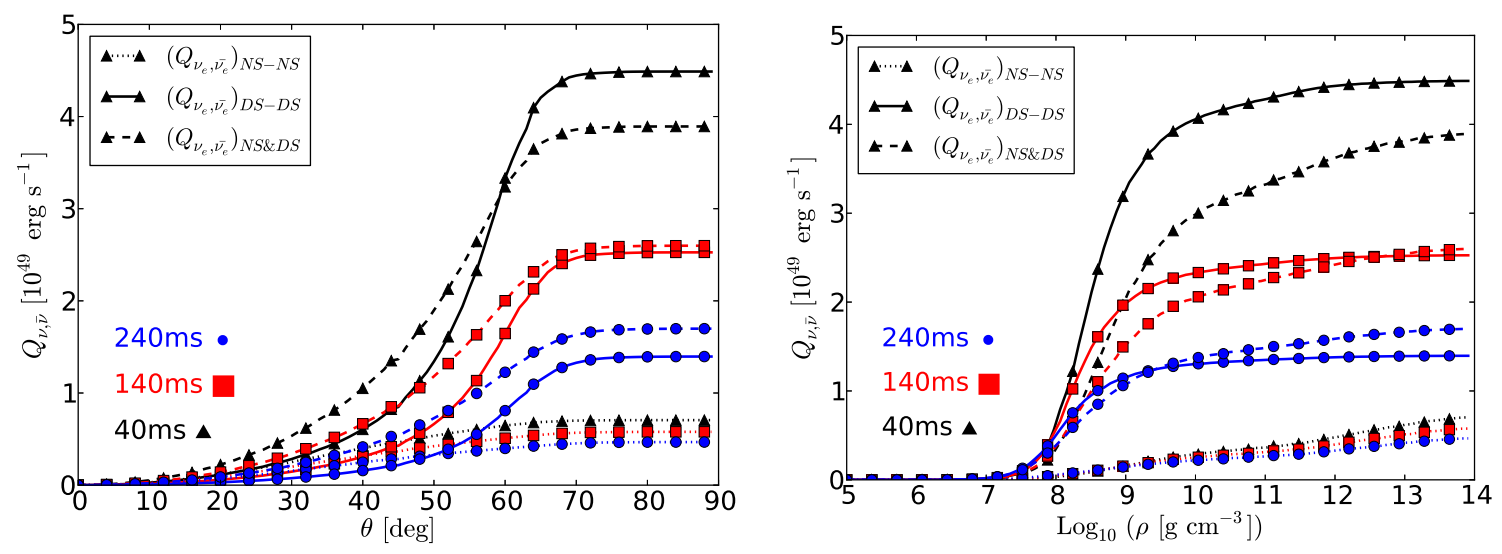

Figure 13. Energy deposition rate by electron neutrino pair annihilation at $40 \mathrm{~ms}$ (black triangles), $140 \mathrm{~ms}$ (red squares) and $240 \mathrm{~ms}$ (blue circles) inside our simulation, as a function of the maximum polar angle (left) and of the maximum matter density where the deposition occurs, for the NS-NS (dotted), DS-DS (solid) and NS\&DS (dashed) contributions. For both panels, we use our standard (Newtonian) results.
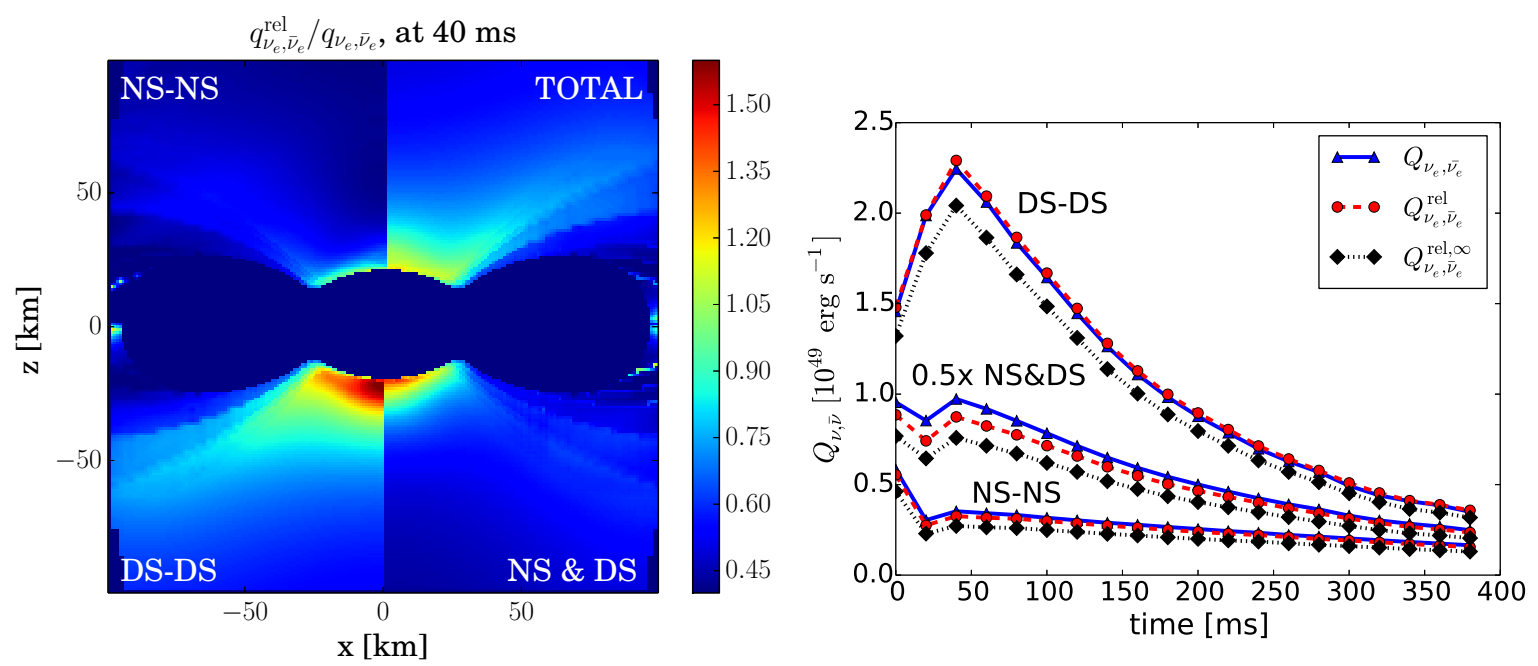

Figure 14. Left: Ratio between the energy deposition rates due to the annihilation of $\nu_{e}, \bar{\nu}_{e}$ pairs, with and without the inclusion of relativistic effects in the neutrino propagation, at $40 \mathrm{~ms}$ inside our simulation. The quantities $q_{\nu_{e}, \bar{\nu}_{e}}$ and $q_{\nu_{e}, \bar{\nu}_{e}}^{\mathrm{rel}}$ are the Newtonian, the general relativistic energy deposition rate, respectively. Each quadrant of the panel represents one of the components of the rate decomposition and their sum. Right: Volume-integrate rates for electron neutrinos, $Q_{\nu_{e}, \bar{\nu}_{e}}$ (blue lines, triangles), $Q_{\nu_{e}, \bar{\nu}_{e}}^{\mathrm{rel}}$ (red lines, circles) and $Q_{\nu_{e}, \bar{\nu}_{e}}^{\mathrm{rel}, \infty}$ (black lines, diamonds), for each of the three contributions (NS-NS, NS\&DS, DS-DS). For the NS\&DS contribution, we plot it halved for visualization purposes.

the latter deposits energies also at larger angular distances from the rotational axis $\left(\theta \gtrsim 45^{\circ}\right)$.

Finally, we show the impact of relativistic effects on the annihilation process. From the left panel of Figure 14, we can infer that the DS-DS contribution is affected the most by relativistic effects. On the one hand, the beaming effect due to the matter motion 

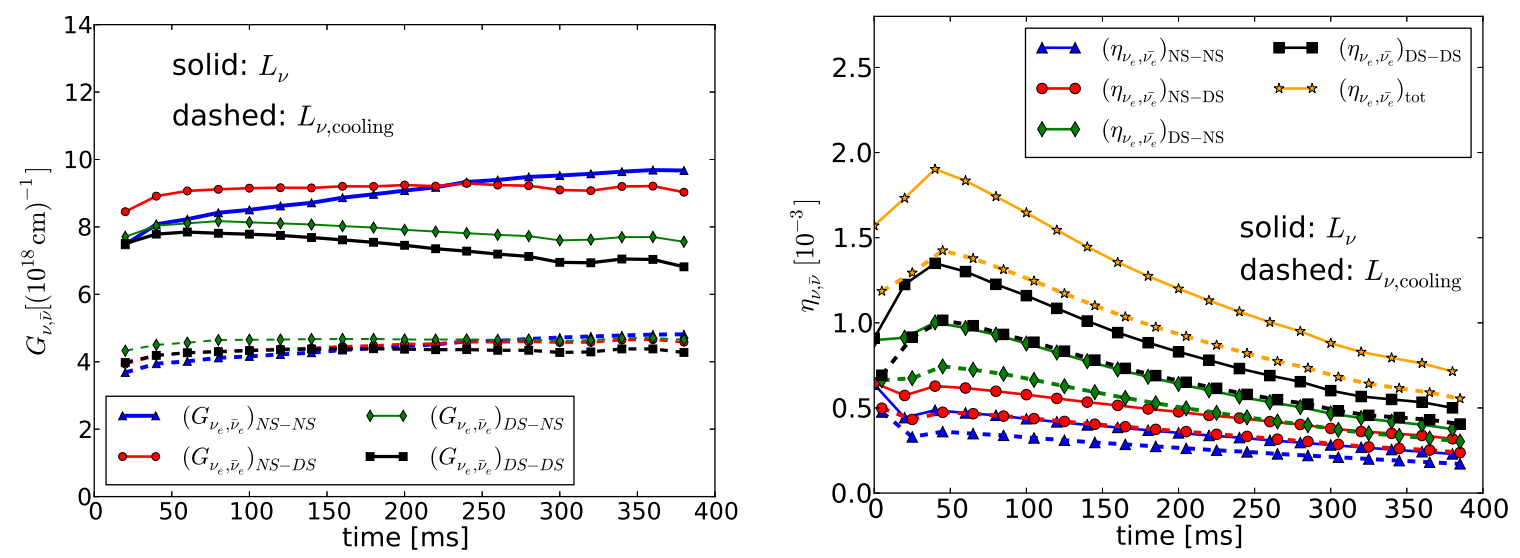

Figure 15. Left: Geometrical factors $G_{\nu, \bar{\nu}}$ (left), defined in Eq. (23), and conversion efficiency parameters $\eta_{\nu, \bar{\nu}}=Q_{\nu, \bar{\nu}} /\left(L_{\nu}+L_{\bar{\nu}}\right)$ (right). Both quantities are calculated for four distinct contributions to the energy deposition rate by $\nu_{e}, \bar{\nu}_{e}$ pairs, using the net luminosities (solid lines) or the cooling luminosities (dashed lines). In the right panel, the dashed lines are shifted by $\Delta t=5 \mathrm{~ms}$ for visualization purposes.

in the disk, together with the Doppler effect and the gravitational redshift, reduces the annihilation rate in the funnel above the MNS. On the other hand, the gravitational blueshift and the light bending occurring close to the MNS and to the innermost neutrino surfaces increase the rates immediately above the MNS surface. The same effects are visible, even if less pronounced, for the NS\&DS contributions. On the contrary, for the NS-NS contribution, the dominant gravitational redshift reduces the annihilation rates almost everywhere. To quantify the global impact of the relativistic effects, we integrate the different contributions over the deposition volume, according to Eqs. (10), (17) and (18). The results are shown in the right panel of Figure 14. In the case of the DSDS contribution, relativistic effects can even marginally increase the integrated energy deposition rate, as measured by local observers, leading to $\left(Q_{\nu, \bar{\nu}}^{\mathrm{rel}}\right)_{\mathrm{DS}-\mathrm{DS}} \approx\left(Q_{\nu, \bar{\nu}}\right)_{\mathrm{DS}-\mathrm{DS}}$. In the case of the NS\&DS and NS-NS contributions, the larger volume and the more pronounced decrease in the energy deposition efficiency at large radii compensate the increase close to the remnant, such that $Q_{\nu, \bar{\nu}}^{\text {rel }} \lesssim Q_{\nu, \bar{\nu}}$. A more detailed analysis of the impact of special and general relativistic effects on the energy deposition rates is presented in Appendix B.

\subsection{Dependence on the neutrino luminosity and conversion efficiency}

In Eq. (12), we have introduced a possible parametrization of the volume-integrated energy deposition rates, $Q_{\nu, \bar{\nu}}$. The advantage of this expression is to provide global information about the energy deposition rate based on basic properties of the neutrino emission $\left(L_{\nu}, L_{\bar{\nu}}\right.$, neutrino mean energies $)$ and on the geometry of the system $\left(G_{\nu, \bar{\nu}}\right.$, $H_{\nu, \bar{\nu}}$. In the following, we verify the validity of our expression and compute the unknown geometrical factors. We take again advantage of the luminosity decomposition and we 
evaluate the geometrical factors separately for each of the contributions appearing in Eq. $(22)$, i.e. $\left(G_{\nu, \bar{\nu}}\right)_{i j}$ and $\left(H_{\nu, \bar{\nu}}\right)_{i j}$ with $i, j=\{\mathrm{NS}, \mathrm{DS}\}$. Since our decomposition distinguishes among contributions presenting different emission properties, we further assume that $\left(G_{\nu, \bar{\nu}}\right)_{i j} \approx\left(H_{\nu, \bar{\nu}}\right)_{i j}$ in Eq. (12) and obtain

$$
\left(Q_{\nu, \bar{\nu}}\right)_{i, j} \approx \frac{\sigma_{0}\left(c_{A}^{2}+c_{V}^{2}\right)_{\nu, \bar{\nu}}}{96 \pi^{2} c\left(m_{e} c^{2}\right)^{2}} L_{\nu, i} L_{\bar{\nu}, j}\left(G_{\nu, \bar{\nu}}\right)_{i, j}\left[\frac{\left\langle\epsilon_{\nu, i}^{2}\right\rangle}{\left\langle\epsilon_{\nu, i}\right\rangle}+\frac{\left\langle\epsilon_{\bar{\nu}, j}^{2}\right\rangle}{\left\langle\epsilon_{\bar{\nu}, j}\right\rangle}\right],
$$

where $\nu, \bar{\nu}$ can be both electron or heavy flavor neutrino pairs. Differently from before, here we distinguish between neutrinos coming from the MNS and antineutrinos coming from the disk, and vice versa, for the $\nu_{e}, \bar{\nu}_{e}$ contributions. In the left panel of Figure 15, we present the temporal evolution of $G_{\nu_{e}, \bar{\nu}_{e}}$ for all the four different contributions to $Q_{\nu_{e}, \bar{\nu}_{e}}$. We tested both the usage of the luminosities at infinity (solid lines), $L_{\nu_{e}}$,

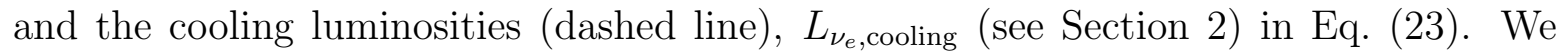
first notice that, despite the different geometrical properties of the MNS and disk emission, the geometrical factors show similar values and similar trends with time, within $10 \%$ of their average values. This result corroborates the validity of parametrization (23). The geometrical factors obtained by the cooling luminosities exhibit a smaller spread, both among them and in time. This reflects the different impact of neutrino absorption outside the neutrino surfaces for the different contributions. In particular, the factor $\sim 2$ between the results computed using $L_{\nu}$ and $L_{\nu \text {,cooling }}$ can be explained as $\left(\exp \left(-\tilde{\tau}_{\nu_{e} \text {,en }}\right) \exp \left(-\tilde{\tau}_{\bar{\nu}_{e}, \text { en }}\right)\right)^{-1}$, where $\tilde{\tau}_{\nu_{e} \text {,en }}$ and $\tilde{\tau}_{\bar{\nu}_{e} \text {,en }}$ are the average values of the energy optical depth at the last scattering surface. Since for $\nu_{e}$, the scattering and the energy optical depth are close (see Figure $(2)), \tilde{\tau}_{\nu_{e}, \text { en }} \approx 0.5 \lesssim 2 / 3$, while for $\bar{\nu}_{e}$ the difference is larger, $\tilde{\bar{\nu}}_{\bar{\nu}_{e} \text { en }} \approx 0.2$.

The situation is qualitatively different for heavy flavor neutrinos (not shown in the figure). Their values are such that $4 \times 10^{-18} \mathrm{~cm}^{-1} \lesssim\left(G_{\nu_{x}, \bar{\nu}_{x}}\right)_{\mathrm{NS}-\mathrm{NS}} \lesssim 5 \times 10^{-18} \mathrm{~cm}^{-1}$, $\left(G_{\nu_{x}, \bar{\nu}_{x}}\right)_{\mathrm{NS}-\mathrm{DS}} \approx 6 \times 10^{-18} \mathrm{~cm}^{-1}$, and $\left(G_{\nu_{x}, \bar{\nu}_{x}}\right)_{\mathrm{DS}-\mathrm{DS}} \approx 7 \times 10^{-18} \mathrm{~cm}^{-1}$. Since there is

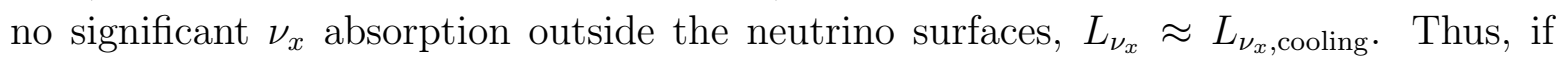
we compare with the results obtained with $L_{\nu_{e} \text {,cooling }}$ and $L_{\bar{\nu}_{e}, \text { cooling }}$, we conclude that the geometrical factors for $\nu_{x}$ are larger. We speculate that the origin of these larger values depends on the smaller extension of the neutrino surfaces. On the one hand, this provides a more isotropic emission, but on the other hand leads to less spatially distributed emission and larger neutrino densities.

After having explored the dependency of the energy deposition rates on the neutrino luminosities, we can quantify the annihilation efficiency, as defined in Eq. (11). In the right panel of Figure 15, we plot $\eta_{\nu_{e}, \bar{\nu}_{e}}$ for the total rates and the four different contributions. In the latter cases, at the denominator, we select only the relevant luminosities, i.e. $\left(\eta_{\nu, \bar{\nu}}\right)_{i, j}=\left(Q_{\nu, \bar{\nu}}\right)_{i, j} /\left(L_{\nu, i}+L_{\bar{\nu}, j}\right)$. Also in this case, we compute $\eta_{\nu_{e}, \bar{\nu}_{e}}$ both using the total (solid lines) and the cooling luminosities (dashed lines), and obviously the former are systematically larger than the latter. The annihilation efficiency of the single components ranges between $0.03 \%$ up to $0.15 \%$. The behavior of the different components and their relative strength results from the non-trivial combinations of neutrino luminosities, geometrical factors, and mean energies. The most 
$\nu-\bar{\nu}$ annihilation above merger remnants: implications of a long-lived MNS

\begin{tabular}{|c|c|cc|cc|cc|}
\hline flavor & contribution & \multicolumn{2}{|c}{ standard } & \multicolumn{2}{|c}{ relativistic (loc) } & \multicolumn{2}{c}{ relativistic $(\infty)$} \\
& & $\left(Q_{\nu, \bar{\nu}}\right)_{0}$ & $t_{0}$ & $\left(Q_{\nu, \bar{\nu}}^{\text {rel }}\right)_{0}$ & $t_{0}$ & $\left(Q_{\nu, \bar{\nu}}^{\text {rel }, \infty}\right)_{0}$ & $t_{0}$ \\
\hline- & - & $10^{49} \mathrm{erg} \mathrm{s}^{-1}$ & $\mathrm{~s}$ & $10^{49} \mathrm{erg} \mathrm{s}^{-1}$ & $\mathrm{~s}$ & $10^{49} \mathrm{erg} \mathrm{s}^{-1}$ & $\mathrm{~s}$ \\
\hline \multirow{5}{*}{$\nu_{e}, \bar{\nu}_{e}$} & $\mathrm{NS}-\mathrm{NS}$ & $3.96(-1)$ & 0.445 & $3.70(-1)$ & 0.450 & $3.08(-1)$ & 0.454 \\
& $\mathrm{DS}-\mathrm{NS}$ & 1.44 & 0.233 & 1.29 & 0.240 & 1.12 & 0.240 \\
& $\mathrm{NS}-\mathrm{DS}$ & $8.86(-1)$ & 0.253 & $7.97(-1)$ & 0.268 & $9.91(-1)$ & 0.268 \\
& $\mathrm{DS}-\mathrm{DS}$ & 2.81 & 0.176 & 2.86 & 0.176 & 2.55 & 0.176 \\
\hline \multirow{3}{*}{$\nu_{x}, \bar{\nu}_{x}$} & $\mathrm{NS}-\mathrm{NS}$ & $8.44(-2)$ & 0.514 & $7.64(-2)$ & 0.513 & $6.41(-2)$ & 0.513 \\
& DS-NS/NS-DS & $3.34(-3)$ & 0.198 & $3.33(-3)$ & 0.205 & $2.88(-3)$ & 0.205 \\
& DS-DS & $1.09(-4)$ & 0.126 & $1.27(-4)$ & 0.128 & $1.12(-4)$ & 0.128 \\
\hline
\end{tabular}

Table 1. Table with the values obtained from the exponential interpolation of the different components of $Q_{\nu, \bar{\nu}}$ (left), $Q_{\nu, \bar{\nu}}^{\mathrm{rel}}$ (center), and $Q_{\nu, \bar{\nu}}^{\mathrm{rel}, \infty}$ (right), for $\nu_{e}, \bar{\nu}_{e}$ (top) and $\nu_{x}, \bar{\nu}_{x}$ (bottom), according to Eq. (24). The integers in bracket correspond to the power of ten of the number.

efficient energy conversion happens for the DS-DS contribution, while the less efficient is the NS-NS contribution. We also compute the efficiency for the total rates (orange line) and find that it varies between $0.2 \%$ (at the beginning, when the annihilation is dominated by the more efficient DS-DS and NS\&DS contributions) and $0.07 \%$ (at the end, when the NS-NS contribution becomes more relevant). We also notice that $\left(\eta_{\nu, \bar{\nu}}\right)_{\text {tot }}$ is larger than any other contribution. This is due to the fact that each component of the luminosity powers two different energy deposition contributions. Thus, we can write $\left(\eta_{\nu, \bar{\nu}}\right)_{\mathrm{tot}}=\sum_{i, j} a_{i, j}\left(\eta_{\nu, \bar{\nu}}\right)_{i, j}$, where $\sum_{i, j} a_{i, j}=2$.

\subsection{MNS collapse time scale}

At the end of our simulation, the annihilation rate has significantly decreased with time, but the cumulative energy deposition has not saturated yet. In addition, we have supposed that the MNS is stable against gravitational collapse on a time scale $>380 \mathrm{~ms}$. In the following, we want to estimate the energy deposition at later time times (1 sec) and investigate the possible impact of $\mathrm{BH}$ formation on the total amount of deposited energy.

We interpolate the different contributions to $Q_{\nu, \bar{\nu}}$ assuming an exponential behavior,

$$
Q_{\nu, \bar{\nu}} \approx\left(Q_{\nu, \bar{\nu}}\right)_{0} \exp \left(-t / t_{0}\right) .
$$

In Table 1, we report the values obtained for the two neutrino species and for the four different contributions in Eq. (22), for our standard case and for calculations including relativistic effects in the neutrino propagation. To account for possible additional energy deposition, we extrapolate our fits for $t>380 \mathrm{~ms}$. We define $t_{\mathrm{BH}}$ as the time when the $\mathrm{BH}$ forms and we assume that for $t>t_{\mathrm{BH}}$ all the contributions to $Q_{\nu, \bar{\nu}}$ involving the MNS $\left(\left(Q_{\nu, \bar{\nu}}\right)_{\mathrm{NS}, \mathrm{NS}}\right.$ and $\left.\left(Q_{\nu, \bar{\nu}}\right)_{\mathrm{NS} \& \mathrm{DS}}\right)$ do not participate in the energy deposition. In Figure 16, we present $\left(E_{\nu, \bar{\nu}}\right)_{\text {ext }}$, the cumulative energy deposition rate extrapolated up 


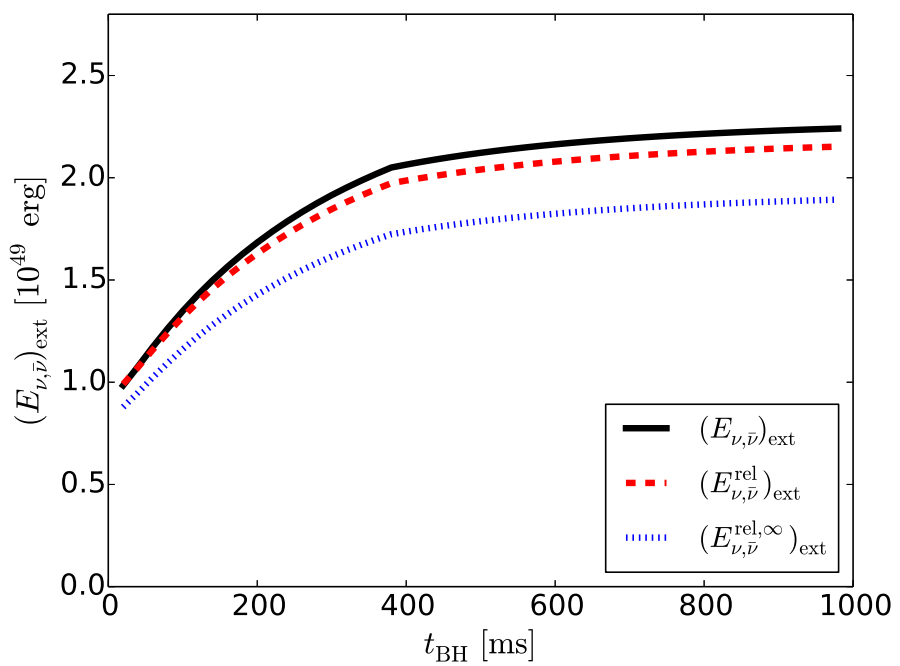

Figure 16. Cumulative energy deposition by all neutrino flavors at 1 sec, as a function of $t_{\mathrm{BH}}$, the collapse time of the MNS to a BH. For $t>t_{\mathrm{BH}}$, contributions of $q_{\nu, \bar{\nu}}$ involving NS neutrinos (NS-NS and NS\&DS) are set to 0. The black solid line represents the reference Newtonian calculations, while the red dashed and blue dotted lines refer to the integral of the local energy and to the energy at infinity, respectively, once relativistic effects in the neutrino propagation have been taken into account.

to one second, as a function of $t_{\mathrm{BH}}$. Since the DS-DS contribution represent $\sim 0.5$ of the total contribution, a quick MNS collapse leads to a reduction of $\lesssim 50 \%$ of the final deposited energy. Due to the decrease of the neutrino luminosities with time, a delayed collapse to a $\mathrm{BH}$ produces a smaller effect on $E_{\nu, \bar{\nu}}$ as time increases. These results are qualitatively insensitive to the modelling of relativistic effects in the neutrino propagation.

\section{Uncertainties on the luminosities and comparison with short GRB energetics}

The results obtained so far rely on calculations of the neutrino luminosities based on one single hydrodynamical model. However, the large variety of initial conditions (NS masses, mass ratios and spins) are expected to translate into a potentially large variety of neutrino luminosities. Moreover, the uncertainties on the nuclear EOS, as well as the impact of relativistic dynamics and different neutrino treatments, can introduce noticeably differences in the intensity of the neutrino luminosities between different numerical models. Specifically, GR merger simulations tends to produce hotter remnant and larger neutrino luminosities (Sekiguchi et al. 2015, Foucart et al. 2016, Radice et al. 2016). A similar effect is observed using softer nuclear EOS. Moreover, gray moment transport schemes seem to suggest lower $\nu_{e}$ luminosities, compared with gray leakage schemes (Foucart et al. 2015). To address the impact of diverse luminosities on the total deposited energy, we employ the proportionality between $L_{\nu} L_{\bar{\nu}}$ and $Q_{\nu, \bar{\nu}}$ in 
$\nu-\bar{\nu}$ annihilation above merger remnants: implications of a long-lived MNS

\begin{tabular}{|c|c|c|c|c|c|c|c|}
\hline label & GRB name & $E_{\gamma, \text { iso }}$ & $E_{\text {kin,iso }}$ & $\theta_{\text {jet,min }}$ & $\theta_{\text {jet,max }}$ & $E_{\text {true,min }}$ & $E_{\text {true,max }}$ \\
\hline- & - & $10^{52} \mathrm{erg}$ & $10^{52} \mathrm{erg}$ & degree & degree & $10^{49} \mathrm{erg}$ & $10^{49} \mathrm{erg}$ \\
\hline $\mathrm{A}$ & $051221 \mathrm{~A}$ & 1.3 & 0.16 & 5 & 8 & 5.55 & 14.21 \\
$\mathrm{~B}$ & $090426 \mathrm{~A}$ & 2.0 & 1.40 & 5 & 7 & 12.94 & 25.34 \\
$\mathrm{C}$ & $111020 \mathrm{~A}$ & 0.17 & 0.48 & 3 & 8 & 0.891 & 6.326 \\
$\mathrm{D}$ & $130603 \mathrm{~B}$ & 0.37 & 0.11 & 4 & 8 & 1.169 & 4.671 \\
\hline $\mathrm{E}$ & 050709 & 0.09 & 0.0026 & 15 & $\sim 30$ & 3.155 & 12.406 \\
$\mathrm{~F}$ & $050724 \mathrm{~A}$ & 0.24 & 0.18 & 25 & $\sim 30$ & 39.35 & 56.27 \\
$\mathrm{G}$ & $101219 \mathrm{~A}$ & 0.74 & 0.30 & 4 & $\sim 30$ & 2.533 & 139.33 \\
$\mathrm{H}$ & $11111 \mathrm{~A}$ & 0.55 & 0.06 & 3 & $\sim 30$ & 0.8359 & 81.72 \\
$\mathrm{I}$ & $120804 \mathrm{~A}$ & 3.4 & 1.10 & 13 & $\sim 30$ & 115.3 & 602.88 \\
$\mathrm{~J}$ & $140903 \mathrm{~A}$ & 0.08 & 2.90 & 6 & $\sim 30$ & 16.32 & 399.24 \\
$\mathrm{~K}$ & $1409030 \mathrm{~B}$ & 0.4 & 0.28 & 9 & $\sim 30$ & 8.372 & 91.10 \\
\hline
\end{tabular}

Table 2. Table with measured short GRB energetics. The isotropized photon and kinetic energies $\left(E_{\gamma, \text { iso }}\right.$ and $\left.E_{\text {kin,iso }}\right)$ are taken from Fong et al. (2015), for their fiducial models $\left(\epsilon_{B}=0.1\right.$ for all cases, but $\epsilon_{B}=10^{-4}$ for $\mathrm{F}$ and but $\epsilon_{B}=10^{-3}$ for $\left.\mathrm{J}\right)$, as well as the jet opening angles. In case only a lower limit for $\theta_{\text {jet,min }}$ is available, an upper limit of $\theta_{\text {jet,max }}=30^{\circ}$ is assumed. The minimal and maximal true energies, $E_{\text {true,min }}$ and $E_{\text {true,max }}$ are computed according to Eq. (25).

Eq. (23), separately for each of the four distinct contributions appearing in Eq. (22). We first assume to rescale separately $L_{\nu}$ and $L_{\bar{\nu}}$, i.e. $L_{\nu} \rightarrow \alpha_{\nu} L_{\nu}$ and $L_{\bar{\nu}} \rightarrow \alpha_{\bar{\nu}} L_{\bar{\nu}}$, by a constant factor $0.5 \leq \alpha_{\nu(\bar{\nu})} \leq 10.0$. In Figure 17, we present the total cumulative deposited energy as a function of $\alpha_{\nu}$ and $\alpha_{\bar{\nu}}$. The four different panels refer to four different $\mathrm{BH}$ formation times and the $\left(\alpha_{\nu}, \alpha_{\bar{\nu}}\right)=(1,1)$ case corresponds to our standard simulation. Luminosities that are twice as large as the computed ones lead to an energy deposition rate four times larger, due to the linear dependence of $Q_{\nu, \bar{\nu}}$ on the product of $L_{\nu}$ and $L_{\bar{\nu}}$. Assuming that neutrino pair annihilation is the only source of energy powering short GRBs and that the deposited energy is converted very efficiently in kinetic energy and photons inside the relativistic jet, we can compare the GRBs inferred energy with the energy computed in our models. Thus, in the same figures, we show also the inferred total energy from the observation of eleven short GRBs, for which also an estimate of the jet opening angle is available, see Table 2, derived from Fong et al. (2015). In four cases (A-D), the temporal steepening of the afterglow decline rate is attributed to jet breaks, and this interpretation provides a measure of the jet opening angle. For the others, the absence of evidences of jet breaks translates in a lower limit for $\theta_{\text {jet }}$, depending on the time of the last observation. In these cases, an upper limit of $\theta_{\text {jet }} \approx 30^{\circ}$ is assumed (Rosswog \& Ramirez-Ruiz 2002). Following Piran (2004), the true energy is computed from the isotropized total energy according to

$$
E_{\text {true }}=\left(1-\cos \theta_{\text {jet }}\right)\left(E_{\gamma, \text { iso }}+E_{\text {kin,iso }}\right) \text {. }
$$



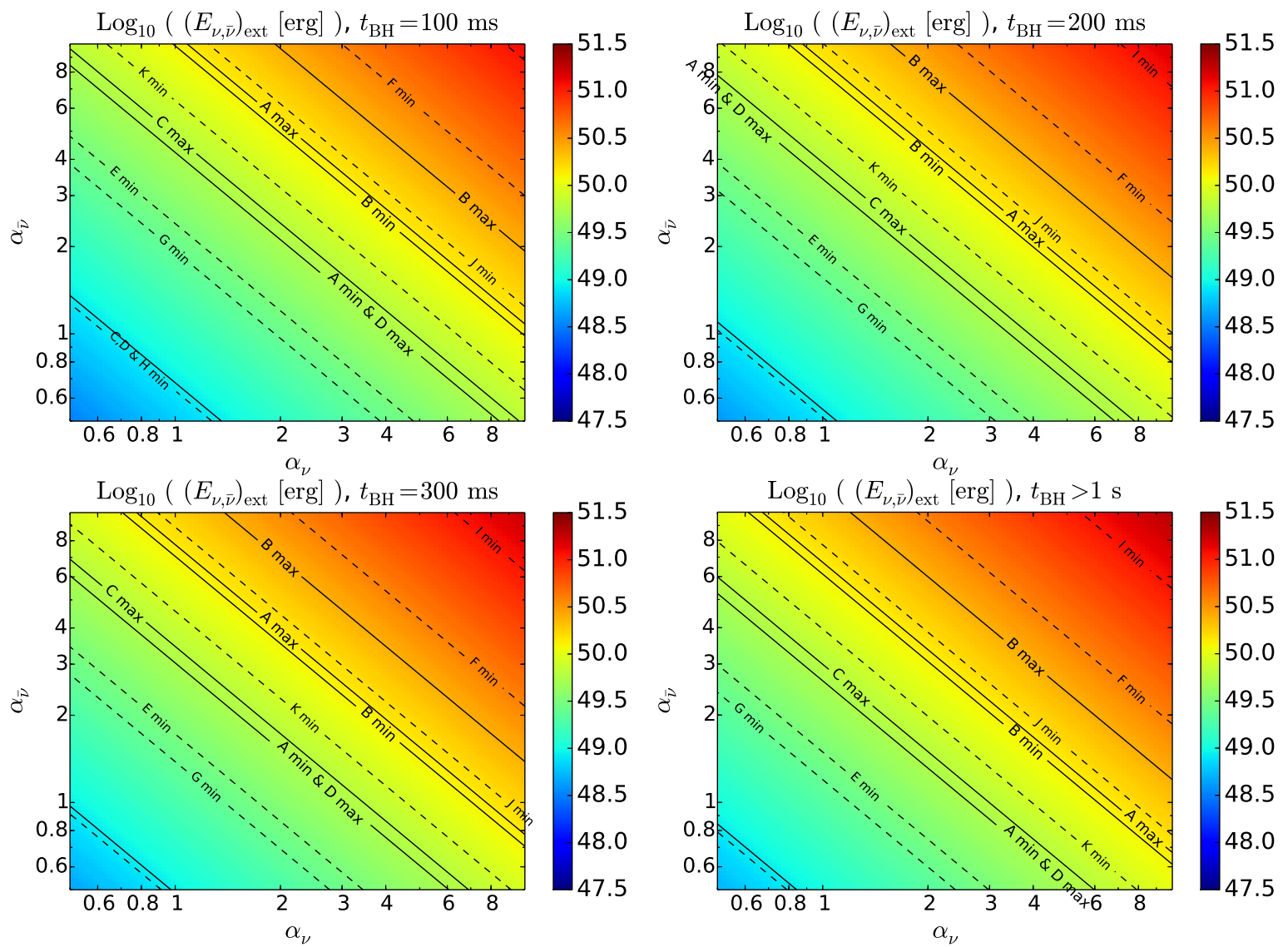

Figure 17. Cumulative energy deposition by $\nu_{e}$ and $\bar{\nu}_{e}$ at $1 \mathrm{~s}$, obtained rescaling the neutrino and the antineutrino luminosities by independent constant factors $0.5 \leq$ $\alpha_{\nu(\bar{\nu})} \leq 10$. The $\alpha_{\nu}=\alpha_{\bar{\nu}}=1$ case the results of our simulation. The four panels refer to different time for the MNS collapse to a BH. Solid lines refer to the inferred minimum and maximum true energy associated with observed short GRBs for which an estimate of the jet opening angle is available. Dashed lines refer to the minimum true energy associated with observed short GRBs for which only lower limit estimates of the jet opening angle are available. GRBs labels, energies and opening angles are detailed in Table 2.

In case the MNS collapses quickly to a $\mathrm{BH}\left(t_{\mathrm{BH}}<100 \mathrm{~ms}\right)$, the results obtained from our simulation are incompatible with all available observations. Also in the case of a long-lived MNS $\left(t_{\mathrm{BH}}>1 \mathrm{~s}\right)$, our results are compatible only with the lower limits of the less energetics GRBs. Significantly larger luminosities $(\alpha \gtrsim 4)$ are needed to explain the energetics of a large fraction of the observed short GRBs. A very delayed collapse to $\mathrm{BH}\left(t_{\mathrm{bh}}>1 \mathrm{~s}\right)$ decreases the required increase in luminosity by a factor $\lesssim 2$, compared with an early collapse $\left(t_{\mathrm{bh}}=100 \mathrm{~ms}\right)$. Nevertheless, the most energetic short GRBs seem to require $\alpha \gtrsim 8$.

We repeat the calculations rescaling the NS and the DS contributions, without distinguishing $\nu_{e}$ from $\bar{\nu}_{e}: L_{\nu, \mathrm{NS}} \rightarrow \alpha_{\mathrm{NS}} L_{\nu, \mathrm{NS}}$ and $L_{\mathrm{DS}} \rightarrow \alpha_{\mathrm{DS}} L_{\nu, \mathrm{DS}}$, and again $0.5 \leq \alpha_{\mathrm{NS}, \mathrm{DS}} \leq 10.0$. In Figure 18, we present the total cumulative deposited energy, extrapolated at one second, as a function of $\alpha_{\mathrm{NS}}$ and $\alpha_{\mathrm{DS}}$, for four different $\mathrm{BH}$ formation 

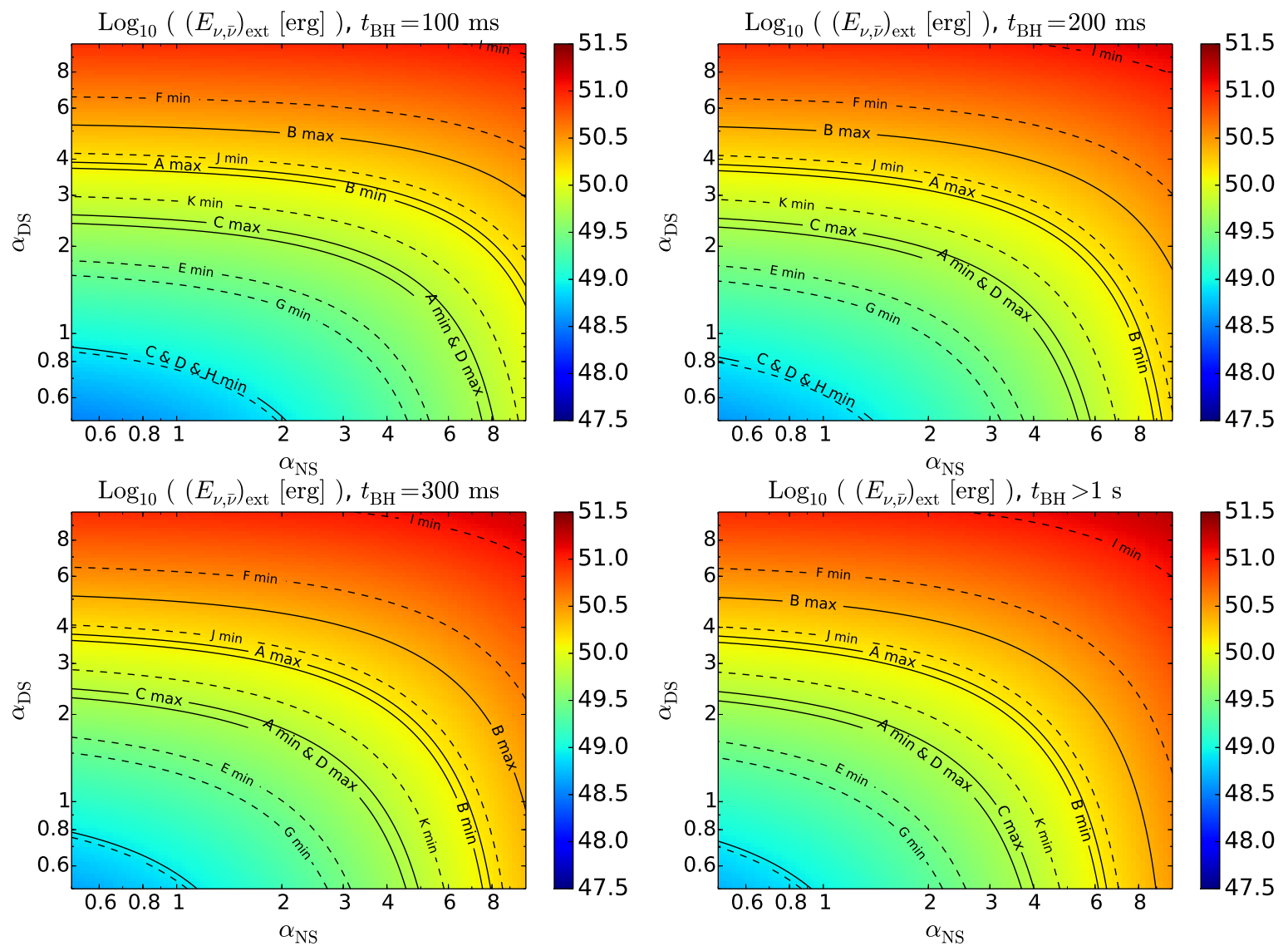

Figure 18. Same as in Figure 17, but rescaling the NS and the DS luminosities by independent constant factors $0.5 \leq \alpha_{\mathrm{NS}, \mathrm{DS}} \leq 10$.

times. Since the NS-NS contribution is smaller than any other contribution involving radiation coming from the disk, there is no more symmetry between $\alpha_{\mathrm{NS}}$ and $\alpha_{\mathrm{DS}}$, and variations along $\alpha_{\text {DS }}$ have a significantly larger impact. Thus, hotter disks and long-lived MNS provide larger energy deposition, and they are necessary ingredients to explain short GRBs energetics with neutrino pair annihilation (at least for bursts of low and medium energy).

\section{Discussion and conclusions}

In this work, we have investigated the energy and momentum deposition operated by the annihilation of $\nu-\bar{\nu}$ pairs above the remnant of a BNS merger over a time scale comparable with the expected disk lifetime ( $400 \mathrm{~ms})$. In particular, we have analyzed the implications of a long-lived MNS and studied the impact of relativistic effects on the neutrino propagation and annihilation. For our study, we have used results of the first long-term, three dimensional simulations of the aftermath of a BNS merger after the influence of neutrino cooling and heating (Perego et al. 2014). The neutrino emission was modeled via a spectral leakage scheme and neutrino pair annihilation rates have been computed outside the neutrino surfaces using a detailed, spectral ray-tracing algorithm. 
Our major findings are:

- the presence of a MNS (instead of a $\mathrm{BH}$ ) in the center increases the annihilation rate by a factor of $\sim 2$, due to the interaction between neutrinos coming from the MNS with antineutrinos coming from the disk, and vice versa. Moreover, it increases the efficiency at which neutrinos can be emitted from the disk, due to the larger emission time and slower accretion process on the MNS surface;

- energy and momentum depositions operated by neutrino pair annihilation are more intense closer to the neutrino surfaces, i.e. immediately above the MNS surface and above the region that marks the transition between the MNS and the disk $\left(q_{\nu, \bar{\nu}} \sim 10^{29} \mathrm{erg} \mathrm{cm}^{-3} \mathrm{~s}^{-1},\left|\mathbf{p}_{\nu, \bar{\nu}}\right| \sim 10^{18} \mathrm{~g} \mathrm{~cm}^{2} \mathrm{~s}^{-2}\right)$. Neutrino annihilation above the MNS occurs at lower polar angles, but larger matter densities, than neutrino annihilation above the innermost part of the disk $(\lesssim 50 \mathrm{~km})$. The location of the annihilation rate and the level of baryonic pollution are expected to influence the dynamical effect of the energy and momentum deposition;

- the net momentum provided by neutrino annihilation is mostly pointing outwards: upwards above the MNS and the densest part of the disk, more radially at large distances from the center. The efficiency at which net momentum is deposited, with respect to the energy deposition, is low close to the remnant $(\lesssim 0.5)$, while efficient $(\gtrsim 0.75)$ far from it;

- the energy and momentum depositions operated by heavy flavor neutrinos are more than one order of magnitude $(\sim 1 / 30)$ smaller than the ones by electron flavor neutrinos;

- volume-integrated energy deposition rates change from $Q_{\nu, \bar{\nu}} \approx 9 \times 10^{49} \mathrm{erg} \mathrm{s}^{-1}$ at the beginning of our simulation to $Q_{\nu, \bar{\nu}} \approx 2 \times 10^{49} \mathrm{erg} \mathrm{s}^{-1}$ at the end (400 ms). They are, in good approximation, proportional to the product of the neutrino luminosities, $L_{\nu} L_{\bar{\nu}}$. Interestingly, the proportionality term that contains all the geometrical dependences of the process $\left(G_{\nu, \bar{\nu}}\right)$ is fairly similar for all the different contributions of $Q_{\nu, \bar{\nu}}$ and rather constant in time;

- the efficiency at which the emitted neutrinos annihilate above the remnant is rather low $\left(\eta_{\nu, \bar{\nu}} \lesssim 0.2 \%\right)$, especially in comparison with the efficiency at which neutrinos are emitted from the disk $\left(\eta_{\text {acc }} \gtrsim 5 \%\right)$;

- the inclusion of relativistic effects in the neutrino propagation does not change the results we have obtained with the Newtonian calculations qualitatively. From a more detailed quantitative analysis, we observe that the beaming of the neutrino radiation emitted from the rotating disk reduces the annihilation rate above the disk and in the funnel; the gravitational redshift further reduces the amount of energy deposited at large distances (a few tens of kilometers) from the remnant, while close to the compact object neutrinos emitted from the disk experience gravitational blueshift and light bending, which increase their energy deposition efficiency, but reduce the momentum deposition one; 
- we computed a cumulative energy deposition of $\approx 2.0 \times 10^{49} \mathrm{erg}$ at $400 \mathrm{~ms}$ and estimated $\approx 2.2 \times 10^{49} \mathrm{erg}$ at $1 \mathrm{~s}$. An early collapse $\left(t_{\mathrm{BH}} \lesssim 100 \mathrm{~ms}\right)$ of the MNS to a $\mathrm{BH}$ decreases the total energy deposition by $\sim 1 / 2$, due to the progressive decrease of the annihilation rate. A late collapse $\left(t_{\mathrm{BH}} \gtrsim 300 \mathrm{~ms}\right)$ is significantly less relevant.

In the following, we compare our results with some recent calculations of neutrino pair annihilation above compact binary mergers in the literature. The initial conditions used in our BNS merger aftermath simulation (Section 2) are very close to the ones used by Dessart et al. (2009). Thus, this work allows a direct comparison, even if our calculations span a much longer time. In their work, Dessart et al. (2009) computed the annihilation rates during the first $100 \mathrm{~ms}$ after the BNS merger using two different approaches: first, a neutrino gray leakage scheme and an annihilation rate formalism based on Ruffert et al. (1996) and Ruffert et al. (1997); second, a $S_{n}$ neutrino transport scheme (Livne et al. 2004, Ott et al. 2008) and a moment formalism for the annihilation rate calculations. Comparing with our results, on the one hand our spatial distribution of the energy deposition rate, Figure 4, is very similar to the one obtained by Dessart et al. (2009) using their second, more accurate approach. On the other hand, significant qualitative differences are visible when comparing with results obtained with their gray leakage scheme. In particular, despite the usage of a leakage scheme, we do not observe a difference of five orders of magnitude between electron and heavy flavor neutrino annihilation rates, rather of a factor 10-100, more similar to the $S_{n}$ results. This comparison reveals that more accuracy can be reached using spectral approaches. Moreover, it confirms that taking into account that neutrinos diffusing from optically thick regions are ultimately emitted from the last scattering neutrino surfaces is key to describe the neutrino intensities and their angular distribution in optically thin conditions (rather than being emitted isotropically from their production site). This effect is more evident for heavy flavor neutrinos, since they decouple much deeper inside the remnant. A more gentle decrease of the total deposited energy with time is observed in our calculations, compared with the steeper decrease found by Setiawan et al. (2006) and Dessart et al. (2009). Consequently, if we integrate the $S_{n}$ results obtained by Dessart et al. (2009) during the first $100 \mathrm{~ms}$, we obtain $\approx 5 \times 10^{48} \mathrm{erg}$, smaller than our result $E_{\nu, \bar{\nu}}(t=100 \mathrm{~ms}) \approx 8 \times 10^{48} \mathrm{erg}$. This is due to the different temporal evolution of the neutrino luminosities between Dessart et al. (2009) and Perego et al. (2014). This difference depends on the diverse neutrino treatments and dimensionality of the two models, as already discussed in Perego et al. (2014). The larger accretion rate obtained in our simulation powers more intense neutrino luminosities, which decrease more gradually than in Dessart et al. (2009).

Richers et al. (2015) computed neutrino annihilation rates in models of compact binary merger remnants using a Monte Carlo radiative transfer code for neutrinos, and comparing with a gray leakage scheme (Metzger \& Fernández 2014). If we restrict the volume integrals of the energy deposition rate only to the two cones of $45^{\circ}$ around the rotational axis, the energy deposited at $400 \mathrm{~ms}$ is $E_{\nu, \bar{\nu}}\left(t=400 \mathrm{~ms}, 45^{\circ}\right) \approx 1.3 \times 10^{49} \mathrm{erg}$, more than six times larger than their results at $3 \mathrm{~s}$ assuming a long-lived MNS 
$\left(1.9 \times 10^{48} \mathrm{erg}\right)$. Since we do not expect the energy deposition to be significantly large for $t>0.5 \mathrm{~s}$, we explain this discrepancy with differences in the two models. In particular, the differences that Richers et al. (2015) discussed in relation with Dessart et al. (2009) apply also to our case. Their MNS is larger, while their disk is significantly smaller (by a factor of 6) and less dense than ours. As a consequence, their optically thin disk produces lower luminosities, and a more spherical and dilute radiation distribution. The different role of the disk in the two models is confirmed by the BH-torus results. In their calculations, the cumulative energy in the $45^{\circ}$ cones at $300 \mathrm{~ms}$ is $2.8 \times 10^{46} \mathrm{erg}$, much lower than the corresponding disk-disk contribution we have computed in our model $\left(E_{\nu, \bar{\nu}}\right)_{\mathrm{DS}-\mathrm{DS}}\left(t=300 \mathrm{~ms}, 45^{\circ}\right) \approx 6.5 \times 10^{48} \mathrm{erg}$.

Our disk-disk contribution can be also compared with the results of Just et al. (2016), where the annihilation of neutrinos above BH-torus systems was computed. Our results at $400 \mathrm{~ms},\left(E_{\nu, \bar{\nu}}\right)_{\mathrm{DS}-\mathrm{DS}}(t=400 \mathrm{~ms}) \approx 8.5 \times 10^{48} \mathrm{erg}$, differ only by $\sim 30 \%$ with the results of their equal mass $\left(1.45 M_{\odot}-1.45 M_{\odot}\right)$ binary merger remnant calculation, despite the usage of different NS masses, EOSs, hydrodynamics and neutrino treatments. In particular, the smaller and faster decreasing neutrino luminosities are compensated by a larger global annihilation efficiency, $\bar{\eta}_{\nu, \bar{\nu}} \approx 0.23 \%$, compared with our results $(\approx 0.13 \%$ at peak).

Finally, we notice that the effects of the inclusion of relativistic effects in the neutrino propagation are qualitatively compatible with results presented in Birkl et al. (2007) and Zalamea \& Beloborodov (2011), for BH-torus calculations. In the latter case, the lack of an extended compact object in the center increases the positive effects close to the $\mathrm{BH}$ horizon and the annihilation rate, compared with Newtonian calculations. Since our hot MNS is rather extended $\left(R_{\mathrm{MNS}} \approx 20 \mathrm{~km}\right)$, these effects are less relevant. For a softer nuclear EOS, we expect a large impact and an increase of the energy deposition rates.

The comparison of the deposited energy we have computed with the inferred energy of observed short GRBs does not support the annihilation of neutrino pairs as a sufficient mechanism to power short GRB jets. Neutrino pair annihilation is still a possible central engine only if the luminosities are significantly larger than the ones we have computed. A factor of 2-3 is usually necessary to explain low energy short GRBs, while a much larger increase $(\gtrsim 8)$ is necessary for the most energetic ones. Smaller jet opening angles $\left(\theta_{\text {jet }} \lesssim 10^{\circ}\right)$ reduce the intrinsic GRB energetics and require smaller luminosity magnification factors. Hotter merger remnants, emerging from general relativistic simulations, possibly employing softer nuclear EOSs, are expected to power significantly larger neutrino luminosities (up to a few times $10^{53} \mathrm{erg} \mathrm{s}^{-1}$ in the first ms after the merger). However, it is unclear if such higher luminosities can be sustained for a long enough time scale. Moreover, general relativistic models predict less massive disks, with presumably smaller lifetimes (e.g., Giacomazzo et al. 2013).

The deposition of an amount of energy compatible with short GRB energetics is not enough to explain the formation of a relativistic jet. The presence of a relatively baryon-free region, where the deposition occurs, is also required to accelerate matter to 
relativistic speeds. Recently, Just et al. (2016) simulated the formation and expansion of jets powered by neutrino pair annihilation in BH-torus systems. They pointed out the possible role of the dynamic ejecta as an obstacle for the formation of a relativistic jet, especially in the case of BNS mergers. On the one hand, a long-lived MNS can potentially pollute more heavily the regions above the remnant via baryon-rich ejecta, in the form of $\nu$-driven (Perego et al. 2014) or magnetically-driven (Siegel et al. 2014) winds. On the other hand, the presence of a large scale $\nu$-driven wind, mainly generated from the disk surface, can collimate the jet within a cone of small opening angle $\left(\theta_{\text {jet }} \lesssim 20^{\circ}\right.$, Murguia-Berthier et al. 2016). Moreover, our results suggest that a longlived MNS increases the amount of deposited energy and net momentum significantly. Nevertheless, the required high luminosities and the difficulties in keeping the funnel above the remnant baryon-free suggest that it is unlikely that neutrino pair annihilation can power the formation of relativistic jets alone. If they still provide the bulk of the jet energy, another mechanism is necessary to keep the funnel free from intense baryon contaminations. Otherwise, an alternative engine is needed and it can work together with neutrino annihilation. If this requires the gravitational collapse of the MNS, the propagation of the jet inside non-relativistic baryon-rich ejecta imposes constraints to the duration of the MNS phase, $t_{\mathrm{BH}} \lesssim 0.4 \mathrm{~s}$, (Murguia-Berthier et al. 2014, MurguiaBerthier et al. 2016).

\section{Acknowledgments}

We thank Raffaella Margutti, Giancarlo Ghirlanda and Gabriele Ghisellini for useful discussions. We acknowledge support from the Helmholtz-University Investigator grant No. VH-NG-825, and from the European Research Council Grant No. 677912 EUROPIUM. This work was also supported by a grant from the Swiss National Supercomputing Centre (CSCS) under project ID 667.

\section{Appendix A. Implementation of relativisitc effects}

In this appendix, we illustrate in detail the implementation of relativistic effects in our annihilation rate calculations.

We model the spacetime outside the MNS as a stationary spherically symmetric spacetime generated by the mass $M_{\mathrm{NS}}$. In the global coordinates $(c t, r, \theta, \phi)$ the metric $g_{\mu \nu}$ is

$$
\mathrm{d} s^{2}=g_{\mu \nu} \mathrm{d} x^{\mu} \mathrm{d} x^{\nu}=-\Gamma^{2}(c \mathrm{~d} t)^{2}+\Gamma^{-2}(\mathrm{~d} r)^{2}+r^{2}(\mathrm{~d} \theta)^{2}+r^{2} \sin ^{2} \theta(\mathrm{d} \phi)^{2},
$$

where $\Gamma=\left(1-2 G M_{\mathrm{NS}} /\left(r c^{2}\right)\right)^{1 / 2}$. Since $M_{\text {disk }} \ll M_{\mathrm{NS}}$, we neglect the effects of the accretion disk mass. We also ignore the effect of the MNS rotation on the spacetime. The spin parameter of the MNS is $\hat{a}_{\mathrm{NS}} \equiv c J_{\mathrm{NS}} /\left(G M_{\mathrm{NS}}^{2}\right) \approx 0.5$, where $J_{\mathrm{NS}}$ is the MNS angular momentum extracted from our simulation. Although the MNS spin is not negligible, $\hat{a}$ is still significantly lower than 1 and $R_{\mathrm{NS}} \gtrsim 2 R_{g}$. Thus, we assume that 
rotational effects on the neutrino propagation do not change the results obtained with the Schwarzschild metric qualitatively.

We compute the energy $\left(q_{\nu, \bar{\nu}}^{\mathrm{rel}}\right)$ and momentum $\left(\mathbf{p}_{\nu, \bar{\nu}}^{\mathrm{rel}}\right)$ deposition rates according to Eqs. (2) and (3), valid in the local stationary frame of each annihilation point. In this locally inertial frame, we can introduce a fixed tetrad $\left\{\mathbf{e}_{\mu}\right\}_{\mu=0 \ldots 3}$, i.e. an orthornormal tetrad fixed with respect to the spatial global coordinates. This basis is defined such that $e_{\mu} \cdot e_{\nu}=\eta_{\mu \nu}$, where $\eta_{\mu \nu}$ is the Minkoswki metric. For the fiducial observer associated with this frame, all the relevant physical quantities are defined as in the flat spacetime. Let's consider neutrinos of energy $\epsilon_{A}$ emitted around a direction $\mathbf{n}_{A}$ from a point $A$ with emissivity $\eta_{A}\left(\epsilon_{A}, \mathbf{n}_{A}\right)$, as measured by the local stationary observer. These neutrinos travel to $B$ (the annihilation point) where they arrive with an energy $\epsilon_{B}$ and a direction $\mathbf{n}_{B}$, as measured by the local stationary observer. The energy at the two points are related by the gravitational redshift formula:

$$
\epsilon_{B}=\left(\frac{\Gamma_{A}}{\Gamma_{B}}\right) \epsilon_{A}
$$

The direction $\mathbf{n}_{B}$ is obtained by solving the geodetic motion of a null particle in the Schwarzschild metric traveling from $A$ to $B$. In Section (Appendix A.1), we detail our method to compute the geodetic motion. Now we want to compute the neutrino intensity at $\mathrm{B}$ based on the emissivity at $A$ : the observer at $B$ measures a number of neutrinos equal to the number of particles emitted at $A$ towards $B$, diminished by the particles absorbed along the propagation path. We estimate the latter quantity similarly to what we have done in the non-relativistic case, Eq. (4):

$$
\mathrm{d} N_{A}\left(1-\exp \left(-\Delta \tau_{\text {en }}\right)\right)=\mathrm{d} N_{B} .
$$

Expressing Eq. (A.3) in terms of the local intensity at $B$ and emissivity at $A$, we obtain

$$
\frac{\eta_{A} \mathrm{~d} V_{A} \mathrm{~d} \Omega_{A} \mathrm{~d} \epsilon_{A} \mathrm{~d} t_{A}}{\epsilon_{A}}=\frac{I_{B} \mathrm{~d} A_{B} \mathrm{~d} \Omega_{B} \mathrm{~d} E_{B} \mathrm{~d} t_{B}}{E_{B}},
$$

and from that

$$
\frac{I_{B} \mathrm{~d} \Omega_{B} \mathrm{~d} E_{B}}{E_{B}}=\left(\frac{\Gamma_{A}}{\Gamma_{B}}\right)\left(\frac{\mathrm{d} \Omega_{A}}{\mathrm{~d} A_{B}}\right) \frac{\eta_{A} \mathrm{~d} V_{A} \mathrm{~d} \epsilon_{A}}{\epsilon_{A}} .
$$

The calculation of $\left(\mathrm{d} \Omega_{A} / \mathrm{d} A_{B}\right)$ is also reported in Section (Appendix A.1).

At the point $A$, the radiation is emitted by matter moving with a velocity $\mathbf{v}_{\mathrm{fl}}=\boldsymbol{\beta} c$, as seen by the stationary observer. The frame comoving with the fluid at $A$ is related to the local stationary frame by a Lorentz boost $\Lambda(\boldsymbol{\beta})$. If we define $\gamma \equiv\left(1-\beta^{2}\right)^{-1 / 2}$, $\cos \psi=\left(\mathbf{p}_{A} \cdot \boldsymbol{\beta}\right) /\left(p_{A} \beta\right), \beta=|\boldsymbol{\beta}|$, and $p_{A}=\left|\mathbf{p}_{A}\right|$, then the energy measured by the local static observer and the emission energy in the comoving frame, $\epsilon_{0}$, are related by the Doppler shift formula:

$$
\epsilon_{A}=\frac{\epsilon_{A, 0}}{\gamma(1-\beta \cos \psi)}
$$

The emissivity transforms between the local and the comoving frames according to

$$
\eta_{A}\left(\epsilon_{A}, \mathbf{n}_{A}\right)=\eta_{A}\left(\epsilon_{A, 0}, \mathbf{n}_{A, 0}\right) \frac{1}{\gamma^{2}(1-\beta \cos \psi)^{3}} .
$$


We notice here that, instead of the usual transformation law for the emissivity, stating that $\eta / \epsilon^{2}$ is a relativistic invariant, we adopted the receiver point of view (e.g., Rybicki \& Lightman 1979), because we express the received intensity at $B$ based on the emissivity at $A$, Eq. (A.6). In this case, the time interval does not transform as $\mathrm{d} t=\gamma \mathrm{d} t_{0}$ (emitter's point of view), but as $\mathrm{d} t=\gamma(1-\beta \cos \psi) \mathrm{d} t_{0}$, due to the Doppler effect. In the comoving frame, the emission is assumed to originate from a volume $\mathrm{d} V_{A, 0}=\gamma \mathrm{d} V_{A}$ and to be isotropic in the half plane defined by a direction $\left\langle\mathbf{n}_{A, 0}\right\rangle$, according to Eq. (6). To relate our relativistic calculations with the input data coming from our Newtonian simulation, we make the following assumptions. First, we assume that Eq. (7) is valid in the comoving reference frame. Second, we assume that the favored emission direction $\mathbf{n}_{\tau}$ of our Newtonian calculations corresponds to $\left\langle\mathbf{n}_{A}\right\rangle$, the average emission direction as seen by the local stationary observer. The sign of the scalar product $\left\langle\mathbf{n}_{A, 0}\right\rangle \cdot \mathbf{n}_{A, 0}$, required in Eq. (6) for Eq. (A.7), is computed using $\mathbf{k}$ and $\mathbf{w}$, two vectors parallel to $\left\langle\mathbf{n}_{A, 0}\right\rangle$ and $\mathbf{n}_{A, 0}$, respectively, and obtained from the Lorentz transformation of the corresponding transformed propagation directions $\left\langle\mathbf{n}_{A}\right\rangle$ and $\mathbf{n}_{A}$ :

$$
\mathbf{k}=\frac{1}{1-\boldsymbol{\beta} \cdot \mathbf{n}_{\tau}}\left[\frac{1}{\gamma} \mathbf{n}_{\tau}-\left(1-\frac{\gamma}{\gamma+1} \boldsymbol{\beta} \cdot \mathbf{n}_{\tau}\right) \boldsymbol{\beta}\right]
$$

and

$$
\mathbf{w}=\frac{1}{1-\boldsymbol{\beta} \cdot \mathbf{n}_{A}}\left[\frac{1}{\gamma} \mathbf{n}_{A}-\left(1-\frac{\gamma}{\gamma+1} \boldsymbol{\beta} \cdot \mathbf{n}_{A}\right) \boldsymbol{\beta}\right]
$$

Thus,

$$
\begin{aligned}
& \operatorname{sign}\left(\left\langle\mathbf{n}_{A, 0}\right\rangle \cdot \mathbf{n}_{A, 0}\right)=\operatorname{sign}(\mathbf{k} \cdot \mathbf{w})=\operatorname{sign}\left(\frac{1}{\left(1-\boldsymbol{\beta} \cdot \mathbf{n}_{\tau}\right)\left(1-\boldsymbol{\beta} \cdot \mathbf{n}_{A}\right)}\right. \\
& \left.\left[1+\left(\boldsymbol{\beta} \cdot \mathbf{n}_{\tau}\right)\left(\boldsymbol{\beta} \cdot \mathbf{n}_{A}\right)-\frac{1}{\gamma^{2}}\left(1-\mathbf{n}_{\tau} \cdot \mathbf{n}_{A}\right)-\boldsymbol{\beta} \cdot \mathbf{n}_{\tau}-\boldsymbol{\beta} \cdot \mathbf{n}_{A}\right]\right) .
\end{aligned}
$$

In a first step, the spectral neutrino fluxes at the annihilation point are computed from the simulation data according to the expressions Eqs. (A.5), (A.7) and (A.10), on an energy grid shifted with respect to the grid used in our hydrodynamics simulation, according to Eqs. (A.2) and (A.6). In a second step, the spectral intensities are interpolated back on the original energy grid. The interpolation is performed in such a way that the energy-integrated neutrino flux is preserved.

\section{Appendix A.1. Geodetic motion}

Here, we clarify the method we have adopted to compute the geodetic motion of a null particle between $A$ and $B$ in the metric (A.1). We first find the rotation $\Psi$ that transforms the global coordinate system to a new coordinate system $\left(c t^{\prime}, r^{\prime}, \theta^{\prime}, \phi^{\prime}\right)$ such that the origin, and the points $A$ and $B$ lie on the plane $\theta^{\prime}=\pi / 2$. Due to angular momentum conservation, the geodetic motion of neutrinos is also confined on this plane. We define $\phi_{A}^{\prime}$ and $\phi_{B}^{\prime}$ as the polar angles of $A$ and $B$ on the $\theta^{\prime}=\pi / 2$ plane, and $\phi_{B}^{\prime}=\phi_{A}^{\prime}-\phi_{A B}$, where $\phi_{A B}$ is the angle between the position vectors of $A$ and $B$ in the original coordinate system. Due to the axisymmetry of the problem around the $z^{\prime}$ 
axis, the choice of $\phi_{A}^{\prime}$ is arbitrary. Since $\Psi$ is a spatial rotation, $r^{\prime}=r$ and $t^{\prime}=t$. The solution of the elliptic integrals that describe exactly the geodetic motion would be computationally too expensive for our annihilation rate calculations. Thus, we use a method derived from the approximated analytic expression provided in Beloborodov (2002). The trajectory of a null particle, expressed in polar coordinates $\left(r^{\prime}, \phi^{\prime}\right)$ on the $\theta^{\prime}=\pi / 2$ plane, can be approximated by

$$
\begin{aligned}
r^{\prime}\left(\phi^{\prime}\right) \approx & {\left[\frac{R_{g}^{2}\left(1-\cos \left(\phi^{\prime}-\Delta\right)\right)^{2}}{4\left(1+\cos \left(\phi^{\prime}-\Delta\right)\right)^{2}}+\frac{b^{2}}{\left.\sin ^{2}\left(\phi^{\prime}-\Delta\right)\right)}\right]^{1 / 2} } \\
& -\frac{R_{g}\left(1-\cos \left(\phi^{\prime}-\Delta\right)\right)}{2\left(1+\cos \left(\phi^{\prime}-\Delta\right)\right)} .
\end{aligned}
$$

In the previous expression, $b$ is the impact parameter, one of the trajectory integrals, and at any point of the trajectory, it can be related to the propagation angle $\alpha$ by

$$
\sin \alpha=\frac{b}{r^{\prime}} \sqrt{1-\frac{R_{g}}{r^{\prime}}} \text {. }
$$

The propagation angle $\alpha$ is defined as the angle between the local propagation direction of the neutrino and the local radial direction, measured in clockwise direction. If we denote $\alpha_{0}$ as the initial propagation angle, the deflection angle $\psi_{0}$ is equal to

$$
\cos \psi_{0}=1-\left(1-\cos \alpha_{0}\right)\left(1-\frac{R_{g}}{r_{A}}\right)^{-1} .
$$

Concerning $\Delta$, if $0 \leq \alpha_{0}<\pi$, then $\Delta=\phi_{A}-\psi_{0}$. In this case, $\phi^{\prime}$ takes values in the interval $\left[\phi_{A}-\psi_{0}, \phi_{A}\right]$, such that $r^{\prime}\left(\phi_{A}\right)=r_{A}$ and $r^{\prime}\left(\phi_{A}-\psi_{0}\right)=+\infty$. On the other hand, if $\pi \leq \alpha_{0}<2 \pi$, then $\Delta=\phi_{A}+\psi_{0}-2 \pi$. In this other case, $\phi^{\prime}$ takes values in the interval $\left[\phi_{A}, \phi_{A}+\psi_{0}\right]$, and $r^{\prime}\left(\phi_{A}\right)=r_{A}$ and $r^{\prime}\left(\phi_{A}+\psi_{0}\right)=+\infty$. The approximated formula replaces the exact elliptic integrals with high accuracy for $r \gtrsim 2 R_{g}$. This approximation is justified because in our case $R_{g} \approx 8 \mathrm{~km}$ and $R_{\mathrm{NS}} \approx 20 \mathrm{~km}$.

Let's assume first that $r_{A}<r_{B}$. Our method consists on the numerical solution of the equation $f\left(\alpha_{0}\right)=0$, where

$$
\begin{aligned}
f\left(\alpha_{0}\right)= & r_{B}-\left[\frac{R_{g}^{2}\left(1-\cos \left(\phi_{B}-\Delta\right)\right)^{2}}{4\left(1+\cos \left(\phi_{B}-\Delta\right)\right)^{2}}+\frac{b^{2}}{\left.\sin ^{2}\left(\phi_{B}-\Delta\right)\right)}\right]^{1 / 2} \\
& +\frac{R_{g}\left(1-\cos \left(\phi_{B}-\Delta\right)\right)}{2\left(1+\cos \left(\phi_{B}-\Delta\right)\right)}
\end{aligned}
$$

obtained by imposing the passage of the trajectory from $A$ and $B$. If a solution of Eq. (A.14) is found, it allows the calculation of the propagation directions $\mathbf{n}_{A}^{\prime}$ and $\mathbf{n}_{B}^{\prime}$ in the rotated local reference frames. In the cases where $r_{B}<r_{A}$, we use the time invariance of the geodetic motion. We solve for the trajectory of a neutrino traveling from $B$ to $A$ and then invert the propagation directions $\mathbf{n}_{\mathrm{inv}} \rightarrow \mathbf{n}_{\mathrm{dir}}=-\mathbf{n}_{\mathrm{inv}}$. Finally, we apply the inverse rotation $\Psi^{-1}$ to these vectors to find the components of $\mathbf{n}_{A}$ and $\mathbf{n}_{B}$ for the original local stationary observers.

The quantity $\left(\mathrm{d} \Omega_{\mathrm{A}} / \mathrm{d} A_{B}\right)$, required in Eq. (A.5), is computed based on our approximated expressions of the trajectory of the null geodesics. In particular, we 

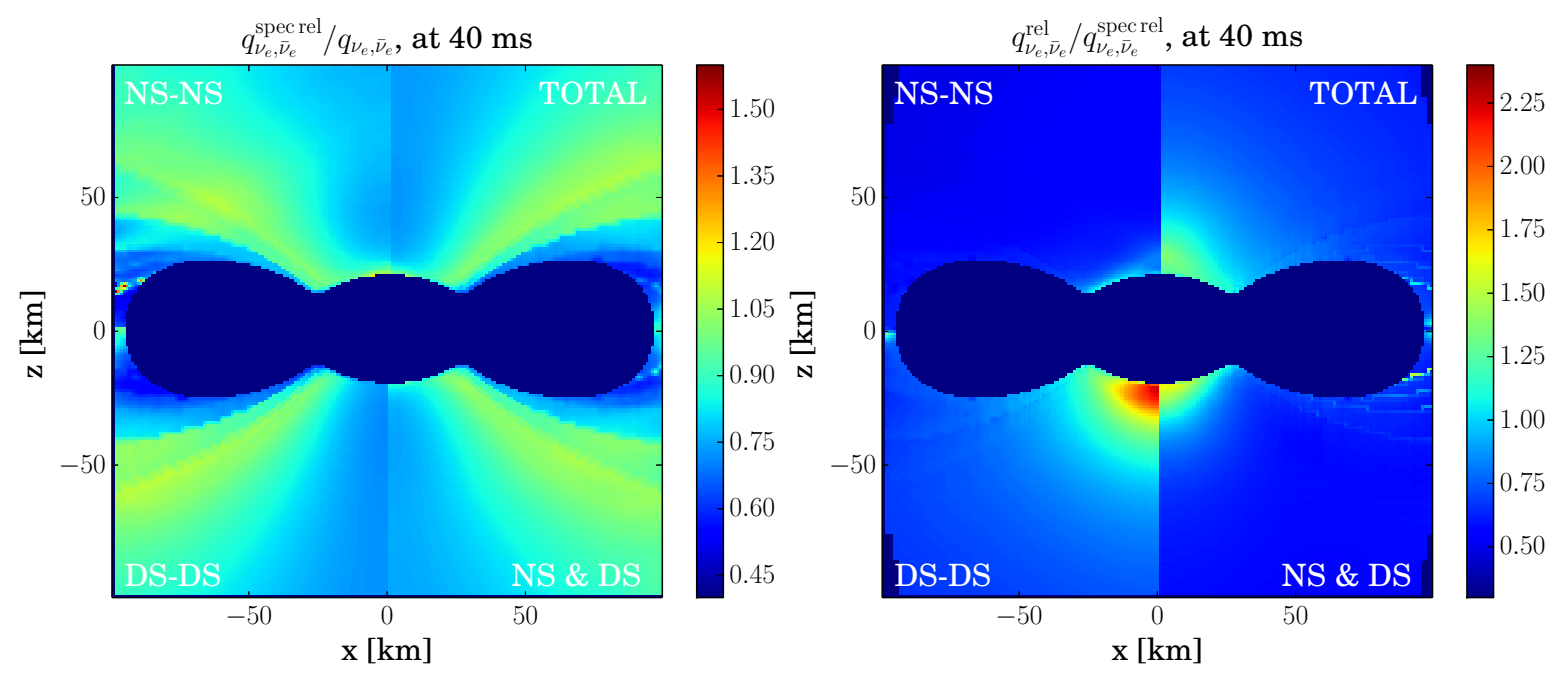

Figure B1. Same as in the left panel of Figure 14, but for $q_{\nu_{e}, \bar{\nu}_{e}}^{\text {spec rel }} / q_{\nu_{e}, \bar{\nu}_{e}}$ (left) and $q_{\nu_{e}, \bar{\nu}_{e}}^{\mathrm{rel}} / q_{\nu_{e}, \bar{\nu}_{e}}^{\mathrm{spec} \text { rel }}$ (right).

consider radiation emitted at $A$ with an initial small spread $\mathrm{d} \alpha_{0}$ in the propagation angle around the direction $\mathbf{n}_{i}\left(\alpha_{0}\right)$, such that $\mathrm{d} \Omega_{A} \approx \pi \mathrm{d} \alpha_{0}^{2}$. This radiation reaches $B$ with a central direction $\mathbf{n}_{f}\left(\alpha_{f}\right)$, within a surface $\mathrm{d} A_{B}=\pi D^{2}$. The quantity $D$ is the length of the perpendicular displacement $\mathrm{d} \mathbf{l}_{B}$, defined such as

$$
\mathrm{d} \mathbf{l}_{B}=-D \sin \alpha_{f} \hat{\mathbf{r}}_{\mathbf{B}}^{\prime}+D \cos \alpha_{f} \hat{\boldsymbol{\phi}}_{\boldsymbol{B}}^{\prime},
$$

assuming to have expressed the final direction as $\mathbf{n}_{f}=\cos \alpha_{f} \hat{\mathbf{r}}_{\mathbf{B}}^{\prime}+\sin \alpha_{f} \hat{\boldsymbol{\phi}}_{\boldsymbol{B}}^{\prime}$. The displacement can also be expressed in terms of the variation of the polar coordinates

$$
\mathrm{d} \mathbf{l}_{B}=\mathrm{d} r_{B}^{\prime} \hat{\mathbf{r}}_{\mathbf{B}}^{\prime}+r_{B}^{\prime} \mathrm{d} \phi_{B}^{\prime} \hat{\boldsymbol{\phi}}_{\boldsymbol{B}}^{\prime} .
$$

If we consider the expression of the trajectory, Eq. (A.14), as a function of both $\phi$ and $\alpha_{0}$, we compute the total differential as:

$$
\mathrm{d} r^{\prime}=\frac{\partial r^{\prime}}{\partial \alpha_{0}} \mathrm{~d} \alpha_{0}+\frac{\partial r^{\prime}}{\partial \phi^{\prime}} \mathrm{d} \phi^{\prime}
$$

Comparing Eqs. (A.15) and (A.16) and using (A.17), we obtain

$$
\left(\frac{\mathrm{d} \Omega_{A}}{\mathrm{~d} A_{B}}\right) \approx\left[\left(-\left.\frac{\partial r^{\prime}}{\partial \phi^{\prime}}\right|_{B} \frac{\cos \alpha_{f}}{r_{f}^{\prime}}-\sin \alpha_{f}\right)\left(\left.\frac{\partial r^{\prime}}{\partial \alpha_{0}}\right|_{B}\right)^{-1}\right]^{2}
$$

\section{Appendix B. Analysis of relativistic effects on the energy deposition rates}

Special and general relativistic corrections have potentially different effects on the four different contributions to the energy deposition rate presented in Eq. (22), due to the different origin and emission geometry. In the color coded panels of Figure B1, we show the ratios between $q_{\nu_{e}, \bar{\nu}_{e}}$ computed with different levels of approximations for the propagation of the neutrinos outside the neutrino surfaces. The rates refer to $40 \mathrm{~ms}$ in our simulation. We consider the standard Newtonian rates, $q_{\nu, \bar{\nu}}$, (Section 3.1), as 
well as the relativistic ones, $q_{\nu, \bar{\nu}}^{\text {rel }}$ (Section 3.2 and Appendix A). As intermediate step, we consider also $q_{\nu, \bar{\nu}}^{\text {spec rel }}$, the energy deposition rate obtained by taking into account only special relativistic effects due to the fast motion of matter inside the remnant (relativistic Doppler and beaming), but neglecting all the relativistic effects due to the curved spacetime (i.e., we assume $\Gamma=1$ in the spacetime metric). The different

quadrants correspond to the different contributions to $q_{\nu_{e}, \bar{\nu}_{e}}$. The ratio $q_{\nu, \bar{\nu}}^{\text {spec rel }} / q_{\nu, \bar{\nu}}$ (left panel) shows that the beaming effect and the Doppler effect reduce the efficiency of the annihilation process significantly in the funnel and, more in general, far from the disk plane, for all the different contributions. At the same time, they increase the energy deposition efficiency close to the remnant. Since neutrinos coming from the disk are more subject to these effects, the largest relative increase is verified for the DS-DS contribution. General relativistic effects modify special relativistic deposition rates mainly in the proximity of the MNS, where the curvature is more pronounced. In particular, the NS-NS contribution benefits only of the radiation bending immediately above the MNS, while in the rest of the volume the redshift decreases the energy deposition rate. For the DS-DS contribution, the better collision angle due to radiation bending and the gravitational blueshift of neutrinos moving towards smaller radii increase significantly the deposition energy efficiency. A similar, although weaker, effect is observed also in the case of the NS\&DS contribution. In this case, neutrinos coming from the MNS are more subject to the gravitational redshift and this explains the faster decrease of the $q_{\nu, \bar{\nu}}$ ratio at larger radii, compared with the DS-DS case.

\section{References}

Aasi J, Abadie J, Abbott B P, Abbott R, Abbott T, Abernathy M R, Accadia T, Acernese F, Adams C, Adams T \& et al. 2015 Classical and Quantum Gravity 32(11), 115012.

Acernese F, Agathos M, Agatsuma K, Aisa D, Allemandou N, Allocca A, Amarni J, Astone P, Balestri G, Ballardin G \& et al. 2015 Classical and Quantum Gravity 32(2), 024001.

Aloy M A, Janka H T \& Müller E 2005 A $\& A$ 436, 273-311.

Asano K \& Fukuyama T 2000 ApJ 531, 949-955.

Aso Y, Michimura Y, Somiya K, Ando M, Miyakawa O, Sekiguchi T, Tatsumi D \& Yamamoto H 2013 PhRD 88(4), 043007.

Bauswein A, Baumgarte T W \& Janka H T 2013 Physical Review Letters 111(13), 131101.

Bauswein A, Goriely S \& Janka H T 2013 ApJ 773, 78.

Beloborodov A M 2002 ApJL 566, L85-L88.

Berger E 2014 ARA $\& A$ 52, 43-105.

Birkl R, Aloy M A, Janka H T \& Müller E 2007 A $\mathscr{\zeta} A$ 463, 51-67.

Blandford R D \& Znajek R L 1977 MNRAS 179, 433-456.

Caballero O L, McLaughlin G C \& Surman R 2012 ApJ 745, 170.

Chen W X \& Beloborodov A M 2007 ApJ 657, 383-399.

Dessart L, Ott C D, Burrows A, Rosswog S \& Livne E 2009 ApJ 690, 1681-1705.

Di Matteo T, Perna R \& Narayan R 2002 ApJ 579, 706-715.

Dionysopoulou K, Alic D \& Rezzolla L 2015 PhRD 92(8), 084064.

Duffell P C, Quataert E \& MacFadyen A I 2015 ApJ 813, 64.

Eichler D, Livio M, Piran T \& Schramm D N 1989 Nature 340, 126-128.

Fernández R \& Metzger B D 2013 MNRAS 435, 502-517. 
Fischer T, Whitehouse S C, Mezzacappa A, Thielemann F K \& Liebendörfer M 2010 A $6 A$ 517, A80. Fong W, Berger E, Margutti R \& Zauderer B A 2015 ApJ 815, 102.

Foucart F, Haas R, Duez M D, O'Connor E, Ott C D, Roberts L, Kidder L E, Lippuner J, Pfeiffer H P \& Scheel M A 2016 PhRD 93(4), 044019.

Foucart F, O'Connor E, Roberts L, Duez M D, Haas R, Kidder L E, Ott C D, Pfeiffer H P, Scheel M A \& Szilagyi B 2015 PhRD 91(12), 124021.

Freiburghaus C, Rosswog S \& Thielemann F 1999 ApJL 525, L121-L124.

Frensel M, Wu M R, Volpe C \& Perego A 2016 ArXiv e-prints .

Ghirlanda G, Salafia O S, Pescalli A, Ghisellini G, Salvaterra R, Chassande-Mottin E, Colpi M, Nappo F, D'Avanzo P, Melandri A, Bernardini M G, Branchesi M, Campana S, Ciolfi R, Covino S, Götz D, Vergani S D, Zennaro M \& Tagliaferri G 2016 A 8 A 594, A84.

Giacomazzo B, Perna R, Rezzolla L, Troja E \& Lazzati D 2013 ApJL 762, L18.

Goodman J, Dar A \& Nussinov S 1987 ApJL 314, L7-L10.

Goriely S, Bauswein A \& Janka H T 2011 ApJL 738, L32+.

Grossman D, Korobkin O, Rosswog S \& Piran T 2014 MNRAS 439, 757-770.

Hempel M, Fischer T, Schaffner-Bielich J \& Liebendörfer M 2012 ApJ 748, 70.

Hotokezaka K, Kiuchi K, Kyutoku K, Muranushi T, Sekiguchi Y i, Shibata M \& Taniguchi K 2013 PhRD 88(4), 044026.

Hüdepohl L, Müller B, Janka H T, Marek A \& Raffelt G G 2010 Physical Review Letters 104(25), 251101.

Janiuk A, Mioduszewski P \& Moscibrodzka M 2013 ApJ 776, 105.

Janka H T 1991 A $\& A$ 244, 378-382.

Jaroszynski M 1993 Acta Astron. 43, 183-191.

Just O, Bauswein A, Pulpillo R A, Goriely S \& Janka H T 2015 MNRAS 448, 541-567.

Just O, Obergaulinger M, Janka H T, Bauswein A \& Schwarz N 2016 ApJL 816, L30.

Kneller J P, McLaughlin G C \& Surman R A 2006 Journal of Physics G Nuclear Physics 32, 443-462.

Korobkin O, Rosswog S, Arcones A \& Winteler C 2012 MNRAS 426, 1940-1949.

Lattimer J M, Mackie F, Ravenhall D G \& Schramm D N 1977 ApJ 213, 225-233.

Li L X \& Paczyński B 1998 ApJL 507, L59-L62.

Livne E, Burrows A, Walder R, Lichtenstadt I \& Thompson T A 2004 ApJ 609, 277-287.

Malkus A, McLaughlin G C \& Surman R 2016 PhRD 93(4), 045021.

Margalit B \& Piran T 2015 MNRAS 452, 3419-3434.

Martin D, Perego A, Arcones A, Thielemann F K, Korobkin O \& Rosswog S 2015 ApJ 813, 2.

Metzger B D \& Fernández R 2014 ArXiv e-prints .

Metzger B D, Giannios D, Thompson T A, Bucciantini N \& Quataert E 2011 MNRAS 413, 2031-2056.

Metzger B D, Martínez-Pinedo G, Darbha S, Quataert E, Arcones A, Kasen D, Thomas R, Nugent P, Panov I V \& Zinner N T 2010 MNRAS 406, 2650-2662.

Miller W A, George N D, Kheyfets A \& McGhee J M 2003 ApJ 583, 833-841.

Murguia-Berthier A, Montes G, Ramirez-Ruiz E, De Colle F \& Lee W H 2014 ApJL 788, L8.

Murguia-Berthier A, Ramirez-Ruiz E, Montes G, De Colle F, Rezzolla L, Rosswog S, Takami K, Perego A \& Lee W H 2016 ArXiv e-prints .

Nagakura H, Hotokezaka K, Sekiguchi Y, Shibata M \& Ioka K 2014 ApJL 784, L28.

Nakar E \& Piran T 2011 Nature 478, 82-84.

Narayan R, Paczynski B \& Piran T 1992 ApJL 395, L83-L86.

Ott C D, Burrows A, Dessart L \& Livne E 2008 ApJ 685, 1069-1088.

Paczynski B 1986 ApJL 308, L43-L46.

Paschalidis V, Ruiz M \& Shapiro S L 2015 ApJL 806, L14.

Perego A, Cabezón R M \& Käppeli R 2016 ApJS 223, 22.

Perego A, Rosswog S, Cabezón R M, Korobkin O, Käppeli R, Arcones A \& Liebendörfer M 2014 MNRAS 443, 3134-3156.

Piran T 2004 Reviews of Modern Physics 76, 1143-1210. 
Popham R, Woosley S E \& Fryer C 1999 ApJ 518, 356-374.

Price D J \& Rosswog S 2006 Science 312, 719-722.

Radice D, Galeazzi F, Lippuner J, Roberts L F, Ott C D \& Rezzolla L 2016 MNRAS 460, 3255-3271.

Raffelt G G 2001 ApJ 561, 890-914.

Richers S, Kasen D, O'Connor E, Fernández R \& Ott C D 2015 ApJ 813, 38.

Roberts L F, Kasen D, Lee W H \& Ramirez-Ruiz E 2011 ApJL 736, L21.

Rosswog S \& Liebendörfer M 2003 MNRAS 342, 673-689.

Rosswog S \& Ramirez-Ruiz E 2002 MNRAS 336, L7-L11.

Rosswog S, Ramirez-Ruiz E \& Davies M B 2003 MNRAS 345, 1077-1090.

Ruffert M \& Janka H T 1999 A\&A 344, 573-606.

Ruffert M, Janka H T \& Schaefer G 1996 A $\& A$ 311, 532-566.

Ruffert M, Janka H, Takahashi K \& Schaefer G 1997 A\&A 319, 122-153.

Rybicki G B \& Lightman A P 1979 Radiative processes in astrophysics.

Sekiguchi Y, Kiuchi K, Kyutoku K \& Shibata M 2015 PhRD 91(6), 064059.

Setiawan S, Ruffert M \& Janka H T 2004 MNRAS 352, 753-758.

Setiawan S, Ruffert M \& Janka H T 2006 A $\& A$ 458, 553-567.

Siegel D M, Ciolfi R \& Rezzolla L 2014 ApJL 785, L6.

Symbalisty E \& Schramm D N 1982 ApJL 22, 143-145.

Tanaka M \& Hotokezaka K 2013 ApJ 775, 113.

Wanajo S, Sekiguchi Y, Nishimura N, Kiuchi K, Kyutoku K \& Shibata M 2014 ApJL 789, L39.

Woosley S E 1993 ApJ 405, 273-277.

Wu M R, Fernández R, Martínez-Pinedo G \& Metzger B D 2016 MNRAS 463, 2323-2334.

Zalamea I \& Beloborodov A M 2011 MNRAS 410, 2302-2308.

Zhu Y L, Perego A \& McLaughlin G C 2016 PhRD 94(10), 105006. 